\title{
Mergers and Acquisitions with Private Equity Intermediation*
}

\author{
Swaminathan Balasubramaniam†, Armando Gomesł SangMok Lee ${ }^{\S}$
}

December 17, 2018

\begin{abstract}
We build a search model of mergers and acquisitions (M\&A) with private equity $(\mathrm{PE})$ intermediation. The model comprises three concurrently operating markets: corporate-corporate, corporate-PE, and inter-PE secondary buyout (SBO). PE intermediaries search for investment opportunities, hold an inventory of firms, add operational value through better governance, and exit by selling their entire portfolio. PE funds can enhance M\&A market efficiency by alleviating search frictions and providing greater liquidity through SBOs, resulting in complementarities among $\mathrm{PE}$ funds. A calibration result shows PE funds' values are higher by $26 \%$ due to SBOs and increasing in the number of funds.
\end{abstract}

Keywords: Mergers and Acquisitions, Private Equity, Secondary Buyouts, OTC Markets, Financial Intermediation

${ }^{*}$ We thank Ana Babus, Paco Buera, Mina Lee, Jason Roderick, and various audiences for helpful comments.

$\dagger^{\dagger}$ Olin Business School, Washington University in St. Louis, Email: balasu.s@wustl.edu

$\ddagger$ Olin Business School, Washington University in St. Louis, gomes@wustl.edu

$\S$ Department of Economics, Washington University in St. Louis, sangmoklee@wustl.edu 


\section{Introduction}

\section{$1.1 \quad$ Overview}

Mergers and acquisitions (M\&A) and private equity (PE) activity contribute to economic efficiency by reallocating assets (such as firms or corporate subdivisions) from low to high productivity corporations (or entrepreneurs) and PE funds. PE buyout funds have become pivotal players in M\&A markets. In 2017, their investment represents $\$ 538$ billion out of the $\$ 2.1$ trillion US M\&A market (or 4,053 out of 10,769 deals) with a compounded annual growth rate of $7.5 \%$ since 2011.1 A typical PE fund (i) acquires a small number of portfolio firms, (ii) holds the firms as inventory and adds operational value through better management, and (iii) exits by selling their portfolio firms to provide liquidity to fund investors. ${ }^{2} \mathrm{PE}$ funds divest the acquired firms primarily to corporate buyers through primary buyouts (PBOs), or to other PE funds through secondary buyouts (SBOs), each of which accounted for $52 \%$ and $42 \%$ of PE exits in 2017. Notably, the share of SBOs in total PE exits has increased significantly from just $13 \%$ in the $1980 \mathrm{~s} \mathrm{~s}^{3}$

We build a search-theoretic M\&A market where PE funds intermediate between corporate sellers and buyers. Our model incorporates unique characteristics of PE funds to a framework similar to Duffie, Gârleanu, and Pedersen (2005). The model allows us to quantify welfare improvement due to M\&A and PE funds and assess the impact of market characteristics, such as search frictions and the number of PE funds, on fund valuations, trading volumes, and transaction prices.

The M\&A market lends itself naturally to a search framework - corporate sellers take about a year to find an appropriate buyer and close a transaction (Boone and Mulherin, 2007). The US M\&A and PE middle market. ${ }^{4}$ which we study in a calibration analysis, is

\footnotetext{
${ }^{1}$ The data on PE activities in the M\&A market is from PitchBook Data, Inc., and can be found at https://pitchbook.com/news/reports/2q-2018-ma-report

${ }^{2}$ We distinguish buyout funds from other PE funds such as venture capital funds, which usually invest in fractional equity stakes of start-ups and early-stage firms.

${ }^{3}$ Initial public offerings (IPOs) are an alternative route for PE funds to divest portfolio firms. However, the share of exit through IPOs is relatively small (6\% of the exits in 2017) and declining (277 per year in 1987-2007 to 98 per year in 2008-2017). Therefore, we omit IPOs from our study.

${ }^{4}$ We define the middle market as corporations with annual revenues between $\$ 20$ million and $\$ 1$ billion, and transactions values between $\$ 25$ million and $\$ 1$ billion, in line with Pitchbook data. See Section 6 for
} 
particularly unintegrated with approximately 100,000 corporations and 2,000 PE funds.

Our model comprises a continuum of corporations and PE funds who hold either one or zero assets, representing firms or corporate divisions, over time. The agents trade assets in one of three markets - (i) corporate- M\&As, (ii) corporate-PE primary buyouts (PBOs), and (iii) inter-PE secondary buyouts (SBOs). In corporate-M\&As, assets are traded from low-type corporations to high-type corporations. High-type corporations can generate higher flow payoffs, due to synergies related to cost reduction or revenue enhancement. Each corporation's type changes over time by an exogenous shock that arrives according to a Poisson process: ${ }^{5}$

On the other hand, PE funds buy assets from low-type corporations and sell assets to high-type corporations through PBOs. PE funds generate a higher flow payoff than low-type corporations due to their better management skill and corporate governance, but a lower flow payoff than high-type corporations because they cannot generate large scale synergies. After purchasing an asset, PE fund investors receive liquidity shocks that arrive according to a Poisson process. Upon receiving a liquidity shock, a PE fund incurs a holding cost from owning assets. The fund then tries to sell the asset either to another PE fund through an $\mathrm{SBO}$ or to a high-type corporation in the PBO market.

We study a steady-state equilibrium in which all three markets are active. We set up a dynamic system of population measures and agents' payoffs, and then prove that a unique steady-state solution exists. We find that PE funds enhance market efficiency by alleviating search frictions and providing greater liquidity to the M\&A market. The first channel, whereby intermediaries facilitate asset transfers and improve efficiency, is well-known. The second channel - the liquidity channel - is unique to our model and is related explicitly to PE funds' demand for liquidity due to their exit requirements.

Our first results focus on the SBO market. SBOs provide greater exit opportunities to $\mathrm{PE}$ funds and enhance PE fund value (Proposition 4) and also improve overall welfare (Proposition 5). SBOs offer a channel through which PE funds can complement each other: funds sourcing deals provide liquidity to funds at the exit phase, and conversely funds at more details.

${ }^{5}$ For example, General Electric became a conglomerate through a spate of acquisitions but has recently announced it will divest its healthcare and oil-service divisions, as it is no longer perceived to be generating the most value with these assets. 
the exit phase allow fund buyers to acquire assets more quickly. The complementarity effect among PE funds could potentially dominate the competition effect. Thus, counterintuitively, PE fund's value can increase in the number of funds, when the number of PE funds is relatively small (Proposition 6). This mechanism can explain why PE funds continue to perform well in spite of the consistently increasing number of $\mathrm{PE}$ funds chasing M\&A deals (Gompers and Lerner, 2000). SBOs are sometimes criticized as opportunistic behavior among fund managers passing sub-par firms to their counterparts while both parties collect management fees (Arcot, Fluck, Gaspar, and Hege, 2015, Wang, 2012) .6 However, our model highlights that it is because of (and not in spite of) SBOs that PE funds generate high returns.

We next consider a fast-search market and obtain a closed-form expression of the equilibrium welfare (Proposition 8). The fast-search equilibrium may not approximate the Walrasian equilibrium and the welfare is subject to search externalities: more search friction in the corporate-M\&A market may increase the welfare. If corporations can transact among themselves quickly, fewer corporate buyers remain in the market. Then, PE funds find it difficult to divest assets, and the exit-phase PE funds suffer the adverse impact of a liquidity shock for a longer time.

A calibration of our model fits well the transaction and price data on the US M\&A and PE middle market and delivers several empirical predictions. The estimated number of tradable assets is larger than the number of high-type corporations or PE funds - which implies that some low-type corporations hold assets and generate only low payoff flows. As expected, the matching rate between PE funds (leading to SBOs) is estimated higher than that between $\mathrm{PE}$ funds and corporations, which in turn is higher than the match intensity between corporations. The value of a PE fund at the investment phase is estimated to be $26 \%$ higher than the value without SBOs. Moreover, as Proposition 6 predicts, this value indeed increases in the number of $\mathrm{PE}$ funds due to the complementarity effect dominating the competition effect. The calibrated model predicts that the share of SBOs over the total $\mathrm{PE}$ fund exits increases with the number of $\mathrm{PE}$ funds, which is consistent with historical evidence.

An increase of PE funds' payoff flow by $1 \%$ increases fund value by $4.2 \%$. If $\mathrm{PE}$ funds

\footnotetext{
${ }^{6}$ See also an article in The Economist at https://www.economist.com/node/15580148.
} 
cannot enhance operating cash flows of acquired firms, then intermediation would not be profitable in spite of their search advantages. The equilibrium welfare is $13.6 \%$ higher than the welfare in the counterfactual situation without M\&A transactions and PE intermediation. Thus, PE funds contribute to the welfare both by lowering search frictions and adding operational value.

\subsection{Related Literature}

To the best of our knowledge, this paper is the first to study PE intermediation in a searchtheoretic M\&A market. In this regard, the current paper is related to several strands of literature: OTC market, M\&A, and the secondary intermediation market.

We build our model based on Duffie, Gârleanu, and Pedersen (2005)'s OTC market, by adding intermediaries that are customized to capture PE funds' characteristics. We discuss only closely related papers on OTC markets with intermediation and refer others to Nosal and Rocheteau (2011) and references therein 77 While PE funds differ from commonly-studied financial intermediaries, some of their features appear in existing papers. Weill (2007) is one of the earliest papers considering intermediaries that hold an inventory. In his model, intermediaries act as buffers of market-wide liquidity shocks, purchasing assets when the market receives a liquidity shock and holding them until the market recovers. In our model, PE funds hold assets in a steady state, rather than just as an impulse response to a shock.

Hugonnier, Lester, and Weill (2018) study an inter-dealer market, which shares some similarities with our PBO and SBO markets. First, dealers in their model have fixed payoff flows over time. Our model is tailored for PE intermediation as PE funds' flow payoffs may change due to liquidity shocks, which yield our new results on SBOs (Propositions 4, 5, and 6). Second, their inter-dealer market is singled out by an assumption that investors can trade only through dealers, which would preclude corporate M\&As in our setting. Our model, with concurrent operation of M\&As, PBOs, and SBOs, allows us to study the influence of the PBOs and SBOs on corporate M\&As and vice versa. This interaction has been empirically documented in a related context by Phillips and Zhdanov (2017), who show that corporate

\footnotetext{
${ }^{7}$ This paper is also related to an extensive literature on the inter-bank network; see a survey by Allen and Babus (2009). We only point out that our model differs from interbank models where the focus is on lending and borrowing.
} 
M\&As incentivize venture capital activities by providing more exit opportunities.

PE funds in our model choose to intermediate between corporate sellers and buyers due to their moderate flow payoff. This self-selection of becoming intermediaries, albeit in different contexts, has also been posited in Neklyudov (2012), Uslu (2016), Nosal, Wong, and Wright (2016), and Farboodi, Jarosch, and Shimer (2017). In these models, it is often the case that mid-type investors, which are similar to our PE funds, choose to intermediate with comparative advantages in search skills 8

In the M\&A literature, Jovanovic and Rousseau (2002)'s "q-theory of mergers" suggests that high-type corporations (i.e., high market-to-book ratio, $q$ ) often acquire low-type corporations to create value. On the contrary, Rhodes-Kropf, Robinson, and Viswanathan (2005) and Rhodes-Kropf and Robinson (2008) find evidence of like-buys-like and explain such assortative matching with buyer-seller complementarities under low search frictions 9 It is worth noting that we are agnostic to the explanations of M\&As. We take M\&A activities as given and instead focus on PE intermediation.

The welfare implications of secondary transactions among intermediaries has been studied in other contexts. Gofman (2014) shows that better-connected intermediaries in financial markets can shorten intermediation chains and improve welfare. Pagano and Volpin (2012) study the bank-loan market where banks lend to consumers (i.e. primary issuance) while other banks provide liquidity by investing in the securitized loans (i.e. secondary market liquidity). They show that high securitization activities in the secondary market yield high loan issuance in the primary market, driven by greater transparency in the secondary market.

Finally, Hochberg, Ljungqvist, and Lu (2007) study the importance of PE funds' networks in sharing information about trading assets. Arcot, Fluck, Gaspar, and Hege (2015), Degeorge, Martin, and Phalippou (2016), and Wang (2012) argue that fund managers under buying pressure initiate SBOs when they are near the end of their investment phase with excess capital, or close to the end of the fund's life. In our paper, SBOs offer a channel

8 Atkeson, Eisfeldt, and Weill (2015) is another study of an OTC market with endogenous intermediation. In an OTC market for derivative swap contracts, investors with risky endowments may be unable to share the risk fully, because of a size limit on bilateral trades. Buy and sell prices do not reflect the aggregate risk, and the price dispersion incentivizes some banks to act as intermediaries.

${ }^{9}$ Other papers relying on q-theory include Eisfeldt and Rampini (2006) and Eisfeldt and Rampini (2008) which study capital reallocations. Also, David (2017) develops a search and matching M\&A model to evaluate the implications of merger activity for aggregate economic outcomes. 
through which PE funds can provide liquidity to each other. We add to the literature on the benefits of secondary transactions by uniquely considering the role of liquidity provision and its interaction with the primary market.

The remainder of the paper is organized as follows. Section 2 introduces the formal model. Section 3 provides equilibrium properties. Section 4 discusses our main analysis of SBOs. Section 5 provides an analysis of the fast-search market, especially the welfare that depends on the search frictions. Section 6 presents a calibration of our model to the US M\&A and PE middle market. Section 7 concludes. All proofs appear in the Appendix.

\section{Model}

Time runs continuously in $t \in[0, \infty)$. Over time, two kinds of agents, corporations and PE funds trade assets, which represent firms or corporate divisions. Initially, a fraction of corporations and PE funds are endowed with tradable assets. The measures of corporations $n_{c}$, PE funds $n_{f}$, and tradable assets $n_{a}$ remain constant ${ }^{10}$ All agents are risk neutral and infinitely lived, with time preferences determined by a constant discount rate $r$. We assume that each agent holds one or zero assets and that $n_{a}<n_{c}$. We normalize the total measure of corporations as $n_{c}=1$.

Each corporation that holds an asset generates either a high payoff flow $u_{h}$ or a low payoff flow $u_{l}\left(<u_{h}\right)$. A corporation does not receive any payoff flow when not holding an asset. The additional flow $u_{h}-u_{l}$ represents some corporations' ability to create synergies via, e.g., cost reductions. A corporation's ability to create payoff flow switches from low to high with Poisson intensity $\rho_{u}$, or from high to low with intensity $\rho_{d}$. The arrival rate of this Poisson shock for each type of corporation is independent of other corporations. The set of corporate types is $\mathcal{T}_{c} \equiv\{h o, l o, h n, \ln \}$, where the letters $h$ and $l$ represent each corporation's ability to generate payoffs, and the letters $o$ and $n$ denote whether a corporation owns an asset or not.

\footnotetext{
${ }^{10} \mathrm{In}$ terms of practice, M\&A transactions include asset purchases, mergers, and acquisitions. While our model states transactions strictly in terms of asset purchases, transactions also include mergers and acquisitions, in which owners of selling firms remain as non-owners in the market and can make further acquisitions.
} 
Each PE fund goes through a life cycle: an investment phase, a harvesting phase, and an exit phase. A fund in the investment phase does not own assets and searches for a corporate or a PE fund selling assets. After purchasing an asset, the fund enters the harvesting phase and creates payoff flow $u_{f}$. A fund in the harvesting phase may sell its assets and start a new life cycle (i.e., goes back to the investment phase), or fund investors receive a liquidity shock with intensity $\rho_{e}$ and the fund enters the exit phase. A fund in the exit phase generates a lower payoff flow $u_{e}\left(<u_{f}\right)$, i.e., it incurs a holding cost until selling the asset. After selling the asset, the fund automatically starts a new life at the investment phase and goes through the cycle as detailed before. We denote a fund in the investing phase by type $f n$ (a fund non-owner), in the harvesting phase by type $f_{o}$ (a fund owner), and in the exiting phase by type $f e$ (a fund that is exiting). The set of fund types is $\mathcal{T}_{f} \equiv\{f n, f o, f e\}$.

We assume that $\mathrm{PE}$ funds in the harvesting phase generate moderate payoff flows, $u_{l}<$ $u_{f}<u_{h}$. PE funds add operational value through better corporate governance (reducing agency costs). However, funds are unable to improve operational value as much as corporate buyers, who can generate greater payoff through synergies. It will turn out later that PE funds, with middle payoff flows, will play the role of intermediaries by purchasing assets from low-type corporations and selling them to high-type corporations. In this sense, we take PE intermediation as given; our objective is to study the unique features of their M\&A activities.

Let $\mathcal{T} \equiv \mathcal{T}_{c} \cup \mathcal{T}_{f}$ denote the set of types with typical elements $i, j$, etc. The measure of type $i \in \mathcal{T}$ at time $t \in[0, \infty)$ is denoted by $\mu_{i}(t)$. Then,

$$
\begin{aligned}
\mu_{h o}(t)+\mu_{h n}(t)+\mu_{l o}(t)+\mu_{l n}(t) & =n_{c}(=1) . \\
\mu_{f n}(t)+\mu_{f o}(t)+\mu_{f e}(t) & =n_{f} . \\
\mu_{h o}(t)+\mu_{l o}(t)+\mu_{f o}(t)+\mu_{f e}(t) & =n_{a} .
\end{aligned}
$$

Agents meet each other over time and negotiate a trade. Two corporations meet each other with intensity $\lambda_{c}>0$ in the corporate-M\&A market. A corporation and a PE fund meet each other with intensity $\lambda_{f}$ in the primary buyout (PBO) market. A PE fund in the exit phase $(f e)$ and a $\mathrm{PE}$ fund in the investment phase $(f n)$ meet each other with intensity $\lambda_{s}$ in the secondary buyout (SBO) market. We assume that the meeting 
rate between any pair of groups is linear in each group's population. That is, for any pair of corporate types $i, j \in \mathcal{T}_{c}$ with measures $\mu_{i}$ and $\mu_{j}$, the total meeting rate is $\lambda_{c} \mu_{i} \mu_{j}$. Similarly, the total meeting rate between a corporate type $i \in \mathcal{T}_{c}$ and a fund type $j \in \mathcal{T}_{f}$ is $\lambda_{f} \mu_{i} \mu_{j}$, and the total meeting rate in the SBO market is $\lambda_{s} \mu_{f e} \mu_{f n}$. When two agents meet each other, they trade an asset instantaneously if and only if the gain from trade (which we explain later) is positive. The assumption of immediate trading upon meeting follows the literature on bargaining without asymmetric information.

We will find an equilibrium in which all submarkets denoted by $\mathcal{M} \equiv\{l o-h n, l o-f n, f o-$ $h n, f e-h n, f e-f n\}$ are active. In the corporate-M\&A market, a lo-type corporation sells an asset to a $h n$-type corporation (lo- $h n$ trade), and the corporations become, respectively, the $l n$-type and ho-type. In the PBO market, either a lo-type corporation sells an asset to a $f n$-type fund ( $l o-f n$ trade), or a PE fund of type either $f o$ or $f e$ sells an asset to a $h n$-type corporation (either $f o-h n$ or $f e-h n$ trade). In the SBO market, a $f e$-type fund sells to a $f n$-type fund ( $f e-f n$ trade). After all trades, the types change from ' $o$ ' to ' $n$ ' and vice versa. Overall, assets are transferred from low-type corporations toward high-type corporations, with a possible chain of trades among PE funds through PBOs and SBOs.

Figure 1 summarizes the model. Agent types are listed on the left column for owners and the right column for non-owners. An owner changes her type to one on the right column upon selling her asset; a non-owner changes her type to one on the left column upon purchasing an asset (a fund's type become fo upon a purchase an asset). The solid arrows represent how assets are transferred from sellers to buyers in the corporate-M\&A market, the PBO market, and the SBO market. The vertical dashed arrows represent the exogenous type changes: high vs. low for corporations, or a liquidity shock to fo-type funds.

We denote an M\&A with PE market by a collection of exogenous variables $\theta \equiv$ $(n, r, u, \rho, \lambda)$, where $n \equiv\left(n_{c}, n_{f}, n_{a}\right), u \equiv\left(u_{l}, u_{h}, u_{f}, u_{e}\right)$, and $\lambda=\left(\lambda_{c}, \lambda_{f}, \lambda_{s}\right)$. When necessary, we also include an initial population distribution $\mu(0)$ in the model. All exogenously given parameters are assumed to be strictly positive ${ }^{11}$

\footnotetext{
${ }^{11}$ We say that a real number $x$ is positive if $x \geq 0$, and strictly positive if $x>0$.
} 


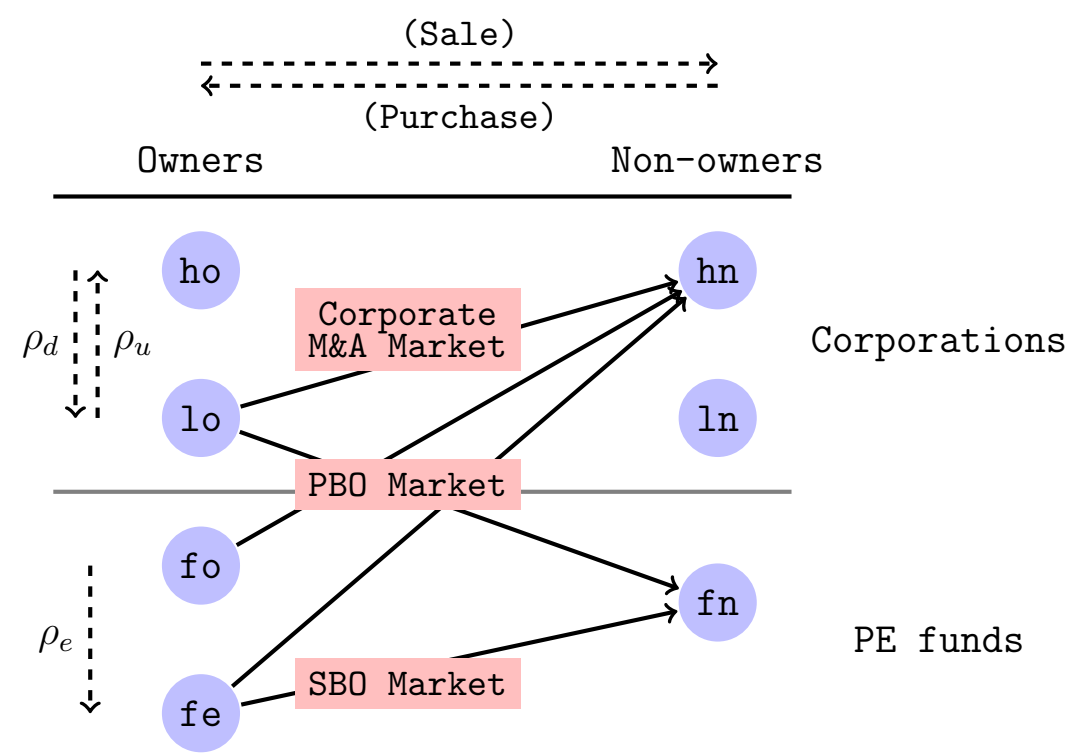

Figure 1: A model of the M\&A with PE market.

\section{Equilibrium}

\subsection{Steady-state equilibrium}

We are interested in a steady-state equilibrium with active trades in all submarkets, a property we aim to have for the calibration exercise in Section 6. That is, in addition to corporations trading assets amongst themselves, $\mathrm{PE}$ funds actively participate in M\&A deals by trading (buying and selling) assets with corporations. Moreover, some exit-phase PE funds sell assets to other PE funds through SBOs. Later, we provide a condition on the market $\theta$ under which such a steady-state equilibrium uniquely exists.

We first derive steady-state population measures for different types. To ease our exposition, we denote the steady-state populations of high- and low-type corporations by $n_{h}$ and $n_{l}$. Since we normalized the total measure of corporations as $n_{c}=1$, from the rates of 
exogenous type changes,

$$
n_{h}=\frac{\rho_{u}}{\rho_{u}+\rho_{d}} \quad \text { and } \quad n_{l}=\frac{\rho_{d}}{\rho_{u}+\rho_{d}}
$$

A $h n$-type corporation switches its type to ho upon purchasing an asset from either a lo-type corporation or a $f_{o-}$ or $f e$-type $\mathrm{PE}$ fund. As such, $h n$-type corporations become $h o$ type at the rate of $\left(\lambda_{c} \mu_{l o}+\lambda_{f} \mu_{f o}+\lambda_{f} \mu_{f e}\right) \mu_{h n}$. On the other hand, as a result of exogenous type changes, $h n$-type corporations switch their types to $l n$ at the rate of $\rho_{d} \mu_{h n}$; similarly, $l n$-type corporations switch their types to $h n$ at the rate of $\rho_{u} \mu_{l n}$. Thus,

$$
\dot{\mu}_{h n}(t)=-\left(\lambda_{c} \mu_{l o}(t)+\lambda_{f} \mu_{f o}(t)+\lambda_{f} \mu_{f e}(t)\right) \mu_{h n}(t)-\rho_{d} \mu_{h n}(t)+\rho_{u} \mu_{l n}(t) . \quad \quad(\mu-\mathrm{hn})
$$

The population measures for other types change over time by similar processes:

$$
\begin{aligned}
& \dot{\mu}_{h o}(t)=\left(\lambda_{c} \mu_{l o}(t)+\lambda_{f} \mu_{f o}(t)+\lambda_{f} \mu_{f e}(t)\right) \mu_{h n}(t)-\rho_{d} \mu_{h o}(t)+\rho_{u} \mu_{l o}(t), \\
& \dot{\mu}_{l n}(t)=\left(\lambda_{c} \mu_{h n}(t)+\lambda_{f} \mu_{f n}(t)\right) \mu_{l o}(t)-\rho_{u} \mu_{l n}(t)+\rho_{d} \mu_{h n}(t), \\
& \dot{\mu}_{l o}(t)=-\left(\lambda_{c} \mu_{h n}(t)+\lambda_{f} \mu_{f n}(t)\right) \mu_{l o}(t)-\rho_{u} \mu_{l o}(t)+\rho_{d} \mu_{h o}(t), \\
& \dot{\mu}_{f n}(t)=\lambda_{f}\left(\mu_{h n}(t) \mu_{f o}(t)+\mu_{h n}(t) \mu_{f e}(t)-\mu_{l o}(t) \mu_{f n}(t)\right), \\
& \dot{\mu}_{f o}(t)=\left(\lambda_{f} \mu_{l o}(t)+\lambda_{s} \mu_{f e}(t)\right) \mu_{f n}(t)-\lambda_{f} \mu_{h n}(t) \mu_{f o}(t)-\rho_{e} \mu_{f o}(t), \\
& \dot{\mu}_{f e}(t)=-\left(\lambda_{f} \mu_{h n}(t)+\lambda_{s} \mu_{f n}(t)\right) \mu_{f e}(t)+\rho_{e} \mu_{f o}(t) .
\end{aligned}
$$

Let $P(\theta)$ denote the above system of population equations $\mu$-hn $-\mu$-fe. A real-vector $\mu \equiv\left(\mu_{i}\right)_{i \in \mathcal{T}}$ with each $\mu_{i} \geq 0$ is a steady-state solution of $P(\theta)$ if the right-hand sides of the equations, with $\mu_{i}(t)$ replaced by $\mu_{i}$ for each $i \in \mathcal{T}$, are equal to zero.

First, we make an important observation:

Lemma 1. If $\mu$ is a steady-state solution of $P(\theta)$, then $\mu_{i}>0$ for all $i \in \mathcal{T}$.

That is, the steady-state population measure of every type is non-vanishing. As a consequence, all kinds of meetings occur with strictly positive rates, so trading can happen in all submarkets (subject to positive gains).

\footnotetext{
${ }^{12}$ Later, a reader can confirm the values of $n_{h}$ and $n_{l}$ by adding population equations $\mu$-ho and $\mu$-hn, or $\mu$-lo and $\mu$-ln , and applying $n_{h}+n_{l}=n_{c}=1$.
} 
The intuition of the lemma is simple. Strictly positive rates $\lambda=\left(\lambda_{c}, \lambda_{f}, \lambda_{s}\right)$ and $\rho=$ $\left(\rho_{u}, \rho_{d}, \rho_{e}\right)$ allow the mass of corporations and PE funds to flow across all types. Given $0<n_{a}<n_{c}+n_{f}$, some fraction of agents are owners and others are non-owners. Corporate owners may have their types changing between high and low exogenously and corporate nonowners have similar probabilistic type changes. As transaction rates $\lambda=\left(\lambda_{c}, \lambda_{f}, \lambda_{s}\right)$ are all strictly positive, some corporations can buy or offload assets after their types are changed. However, not all of the corporations can do so within any fixed time period. A similar idea holds for PE funds.

We show that a unique steady-state solution of $P(\theta)$ exists and it is asymptotically stable: if the population measure $\mu(0)$ is perturbed locally from the steady-state solution $\mu$, then the measure $\mu(t)$ gradually converges back to $\mu$.

Proposition 1. (Steady-state Population Measures) Take any MEA A with PE market $\theta$.

1. (Existence and Uniqueness) There exists a unique steady-state solution $\mu$ of $P(\theta)$.

2. (Asymptotic Stability) Let $\mu(t)$ be a dynamic solution of $P(\theta)$ with initial condition $\mu(0)$. For any $\epsilon>0$, there exists $\delta>0$ such that, if $\|\mu(0)-\mu\|<\delta$, then $\|\mu(t)-\mu\| \leq \epsilon$ for all $t$, and $\mu(t) \rightarrow \mu$ as $t \rightarrow \infty$.

We prove the existence and uniqueness of a steady-state solution by the Poincare-Hopf index theorem (Simsek, Ozdaglar, and Acemoglu, 2007), which is new to the literature on intermediated OTC markets 13

To get an intuition, we set $\left(\mu_{i}\right)_{i \in \mathcal{T}_{c}} \approx 0$, while satisfying the population equations but violating $n_{c}=1$. A small increase of $\mu_{l o}$ (or $\mu_{h n}$ ) increases the supply (resp., demand) of assets in the corporate-M\&A market, the number of M\&A deals $\lambda_{c} \mu_{l o} \mu_{h n}$, and in turn the number of corporations that are rightly holding or not-holding assets $\left(\mu_{h_{o}}\right.$ and $\left.\mu_{l n}\right)$. This increased populations of $\mu_{h o}$ and $\mu_{l n}$ lead to more inflow $\rho_{d} \mu_{h o}$ of agents back to the aggregate supply and the inflow $\rho_{u} \mu_{l n}$ to the aggregate demand in the corporate-M\&A market. That

\footnotetext{
${ }^{13}$ The uniqueness of a steady-state equilibrium is not universal in the literature on OTC, or more broadly search-and-bargain, markets with intermediation. A unique steady-state equilibrium appears in Duffie, Gârleanu, and Pedersen (2005), but multiple equilibria appear more commonly with financial market applications: e.g., Vayanos and Weill (2008) and Trejos and Wright (2016).
} 
is, all four corporate-type populations increase. We take into account how corporate-type populations are related to fund-type populations (which is elaborated in Subsection A.2) and show that a unique supply $\mu_{l o}$ and demand $\mu_{h n}$ satisfy the constraint $\mu_{l o}+\mu_{h n}+\mu_{l n}+\mu_{h o}=$ $n_{c}=1$, by the index theorem, which generalizes the Intermediate Value Theorem. The second part of the proposition on stability is due to a classical result in dynamical systems (Hirsch and Smale, 1973): if all eigenvalues of the linearized system at the steady-state solution have negative real parts, then the solution is asymptotically stable.

We define a steady-state equilibrium via a recursive equation of certain values. The sources of value to all agents in our model are two-fold: (i) flow payoffs for the duration of assets held and (ii) gains from trade. Let $v_{h n}$ denote the expected value of time-discounted future payoffs for a type- $h n$ corporation. The value is defined implicitly by

$$
r v_{h n}=\lambda_{c} \mu_{l o} g_{l o-h n}+\lambda_{f} \mu_{f o} g_{f o-h n}+\lambda_{f} \mu_{f e} g_{f e-h n}-\rho_{d}\left(v_{h n}-v_{l n}\right),
$$

where each $g_{l o-h n}, g_{f o-h n}$, and $g_{f e-h n}$ denotes the corporation's gain from trade (in fact, an equal share of the gain, which we will define later) in each submarket. The rate of meeting a new potential trade partner in the corporate-M\&A market, taking into account the population of sellers, is $\lambda_{c} \mu_{l o}$, and the gain from a trade is $g_{l o-h n}$. Two other terms are defined similarly for the cases of trading in the PBO market with either a fo- or fe-type PE fund. The corporation changes its type from high to low with rate $\rho_{d}$, in which case, it loses value equivalent to $v_{h n}-v_{l n}$.

The values for other types are similarly defined implicitly as follows:

$$
\begin{aligned}
r v_{h o} & =u_{h}-\rho_{d}\left(v_{h o}-v_{l o}\right), \\
r v_{l n} & =\rho_{u}\left(v_{h n}-v_{l n}\right), \\
r v_{l o} & =u_{l}+\lambda_{c} \mu_{h n} g_{l o-h n}+\lambda_{f} \mu_{f n} g_{l o-f n}+\rho_{u}\left(v_{h o}-v_{l o}\right), \\
r v_{f n} & =\lambda_{f} \mu_{l o} g_{l o-f n}+\lambda_{s} \mu_{f e} g_{f e-f n}, \\
r v_{f o} & =u_{f}+\lambda_{f} \mu_{h n} g_{f o-h n}-\rho_{e}\left(v_{f o}-v_{f e}\right), \\
r v_{f e} & =u_{e}+\lambda_{f} \mu_{h n} g_{f e-h n}+\lambda_{s} \mu_{f n} g_{f e-f n} .
\end{aligned}
$$

A term representing flow payoffs is included for each owner type (the payoff flow is zero for 
non-owners).

We assume that buyers and sellers in each submarket have equal bargaining power ${ }^{14}$ That is, the transaction prices (that we will characterize in the next subsection) will be set to ensure an equal division of gain from trade between a buyer and a seller. Each trading partner's gain is:

$$
\begin{aligned}
g_{l o-h n} & \equiv(1 / 2)\left(v_{h o}+v_{l n}-v_{l o}-v_{h n}\right), \\
g_{l o-f n} & \equiv(1 / 2)\left(v_{f o}+v_{l n}-v_{l o}-v_{f n}\right), \\
g_{f o-h n} & \equiv(1 / 2)\left(v_{h o}+v_{f n}-v_{f o}-v_{h n}\right), \\
g_{f e-h n} & \equiv(1 / 2)\left(v_{h o}+v_{f n}-v_{f e}-v_{h n}\right), \\
g_{f e-f n} & \equiv(1 / 2)\left(v_{f o}+v_{f n}-v_{f e}-v_{f n}\right)=(1 / 2)\left(v_{f o}-v_{f e}\right) .
\end{aligned}
$$

Let $V(\theta)$ denote the above system of value equations $(\mathrm{v}-\mathrm{hn})-(\mathrm{v}-\mathrm{fe})$, with $\mu$ being replaced by the unique steady-state solution of $P(\theta)$. The following proposition ensures that the values $v \equiv\left(v_{i}\right)_{i \in \mathcal{T}}$ are well-defined:

Proposition 2. There exists a unique solution of $V(\theta)$.

We have so far characterized the steady-state population measures and the values, assuming that all submarkets are active. We now define a steady-state equilibrium as a pair $(\mu, v)$ such that $\mu$ is a steady-state solution of $P(\theta), v$ solves $V(\theta)$, and the gains from trade are positive:

Proposition 3. A steady-state equilibrium, with all submarkets being active, exists if and only if (19) and (20) in Subsection A.5 hold. Whenever a steady-state equilibrium exists, it is unique.

Gains from trades in certain submarkets are easily positive. For example, $g_{f e-f n} \geq 0$ is immediate from $u_{f}>u_{e}$ : SBOs bail out PE funds under liquidity constraints. The gains in the corporate-M\&A market and the PBO market are related as $g_{l o-h n}=g_{l o-f n}+g_{f o-h n}$ : a direct $M \& A$ and an indirect M\&A, through PE intermediation, result in the same total gains.

\footnotetext{
${ }^{14}$ Our qualitative results do not depend on this assumption. In fact, Ahern (2012) observes that the dollar gains of M\&A deals are often equally split between buyers and sellers.
} 
In a similar vein, gains in the PBO and SBO markets are related as $g_{f e-h n}=g_{f o-h n}+g_{f e-f n}$. Therefore, it remains to make sure that two gains $g_{l o-f n}$ and $g_{f o-h n}$ are positive, which corresponds, respectively, to (19) and 20).

These two gains are obviously positive if differences between payoff flows $u_{f}-u_{l}$ and $u_{h}-u_{f}$ are sufficiently large. We obtain a simple expression for 200 when $\rho=\left(\rho_{u}, \rho_{d}, \rho_{e}\right)$ is close to zero ${ }^{15}$ We consider the case of $n_{a}>n_{h}$ (else, given rare exogenous type changes, almost all assets will be held by high-type corporations and there will be little to no trade). Then, $\mu_{h n} \approx 0$, as there are hardly any type changes from $l n$ to $h n$, and ensuring $g_{l o-f n} \geq 0$ becomes equivalent to:

$$
u_{f}+0 \geq u_{l}+\lambda_{s} \mu_{f e} g_{f e-f n} .
$$

Each side of the inequality considers a steady-state in which corporate sellers $(l o)$ and fund buyers $(f n)$ either trade or do not trade. On the left-hand side (i.e., trading in steady state), the total payoff flows after trading is likely to be $u_{f}$ for a long time; a liquidity shock rarely arrives, and corporate buyers $(h n)$ for PE funds' assets are scarce. On the right-hand side (i.e., no trading in steady state), an lo-type corporation receives payoff flow $u_{l}$ for an extended period of time, as she does not trade with a fund buyer $(f n)$ and the corporate buyer $(h n)$ are scarce. Instead, a fund buyer $(f n)$ will purchase an asset from an exiting fund $(f e)$ with the gain $g_{f e-f n}$.

The above condition for positive gains always holds under overall scarcity of tradable assets (i.e., $n_{h}+n_{f}>n_{a}$ ). Then, $\mu_{f e} \approx 0$ because a non-negligible number of $f n$-type funds try to purchase assets, partly from $f e$-type funds, whereas liquidity shocks hardly arrive to fo-type funds. If the market has a surplus of tradable assets (i.e., $n_{a}>n_{h}+n_{f}$ ), then the condition holds if $\mathrm{PE}$ funds make a significant operational improvement (i.e., a large $\left.u_{f}-u_{l}\right)$. Notice that $\mu_{f e} \leq n_{f}$ and $g_{f e-f n} \approx \frac{u_{f}-u_{e}}{2 r}$, as the total gains from each SBO $\frac{u_{f}-u_{e}}{r}$ is equally shared between buyers and sellers. Therefore, a simple expression for 20 is $u_{f}-u_{l} \geq \lambda_{s} n_{f}\left(\frac{u_{f}-u_{e}}{2 r}\right)$.

\footnotetext{
${ }^{15} 200$ implies (19), when $\lambda_{c}<\lambda_{f}<\lambda_{s}$ and $\rho \rightarrow 0$. For a proof, we take $\rho \rightarrow 0$ in (18) (where $g_{1} \equiv g_{f o-h n}$ and $\left.g_{2} \equiv g_{l o-f n}\right)$ and show that $g_{l o-f n} \geq 0$ implies $g_{f o-h n} \geq 0$. The inequalities over meeting rates are reasonable assumptions for the $\mathrm{M} \& \mathrm{~A}$ with $\mathrm{PE}$ market and also turn out to be the case in our calibration in Section 6
} 


\subsection{Transaction prices}

In a steady-state equilibrium, the transaction prices in all submarkets are determined in a manner so that buyers and sellers equally share the gains from trades (the equal bargaining power assumption). For example, in the corporate-M\&A market,

$$
p_{l o-h n} \equiv(1 / 2)\left(v_{h o}+v_{l o}-v_{h n}-v_{l n}\right),
$$

so that a buyer's gain $\left(v_{h o}-p_{l o-h n}\right)-v_{h n}$ and a seller's gain $\left(v_{l n}+p_{l o-h n}\right)-v_{l o}$ are the same. The transaction prices for other submarkets are similarly determined 16

PE funds derive profits from two sources: (i) operating cash flows of portfolio firms and (ii) the spread on investment and divestments of portfolio firms. We establish below various relationships among transaction prices and spreads:

Lemma 2. (Equilibrium Prices)

1. $p_{f o-h n} \geq p_{f e-h n} \geq p_{f e-f n}$ : PE funds sell at a lower price, if they sell during the exit phase rather than in the harvesting phase, and at an even lower price, if they sell through SBOs.

2. $p_{f o-h n} \geq p_{l o-h n} \geq p_{l o-f n}$ : When selling assets, PE funds' prices are higher than in the corporate-MEA market; when purchasing assets, PE funds pay less than in the corporate-MEA market.

A PE fund that manages to sell assets before receiving liquidity shocks can generate positive profits, from payoff flows $u_{f}$ and the positive spread $p_{f o-h n}-p_{l o-f n}$. If a PE fund suffers a liquidity shock before finding a corporate buyer, the fund may incur losses ex-post as the spread at the exit phase $p_{f e-h n}-p_{l o-f n}$ can be negative.

\subsection{Welfare}

In the M\&A with PE market, assets are reallocated from low-type corporations to high-type corporations and PE funds. We introduce several welfare measures to quantify the economic

\footnotetext{
${ }^{16}$ To be more precise, $p_{l o-f n}=(1 / 2)\left(v_{f o}+v_{l o}-v_{l n}-v_{f n}\right), p_{f o-h n}=(1 / 2)\left(v_{h o}+v_{f o}-v_{f n}-v_{h n}\right)$, $p_{f e-h n}=(1 / 2)\left(v_{h o}+v_{f e}-v_{f n}-v_{h n}\right)$, and $p_{f e-f n}=(1 / 2)\left(v_{f o}+v_{f e}-2 v_{f n}\right)$.
} 
effects of the reallocation.

Given a steady-state equilibrium $(\mu, v)$, the aggregate value of all agents is given by $\sum_{i \in \mathcal{T}} \mu_{i} v_{i}$. The corporate sector's contribution is $\sum_{i \in \mathcal{T}_{c}} \mu_{i} v_{i}$, and the financial sector's contribution is $\sum_{i \in \mathcal{T}_{f}} \mu_{i} v_{i}$. The aggregate value must be naturally related to the steady-state (surplus) welfare, defined by

$$
W \equiv \mu_{h o} u_{h}+\mu_{f o} u_{f}+\mu_{f e} u_{e}+\mu_{l o} u_{l}
$$

The relation between the aggregate values - of all agents, corporations, and PE funds - and the welfare are as follows:

Lemma 3. Take any market $\theta$ with a unique steady-state solution $(\mu, v)$. Then, the welfare and the aggregate values are related by

$$
W=r \sum_{i \in \mathcal{T}} \mu_{i} v_{i}, \quad W_{c}=r \sum_{i \in \mathcal{T}_{c}} \mu_{i} v_{i}, \quad \text { and } \quad W_{f}=r \sum_{i \in \mathcal{T}_{f}} \mu_{i} v_{i}
$$

where the welfare of corporations and PE funds are respectively,

$$
\begin{aligned}
& W_{c} \equiv \mu_{h o} u_{h}+\mu_{l o} u_{l}+\underbrace{\lambda_{f} \mu_{l o} \mu_{f n} p_{l o-f n}}_{\text {sales to PE funds }}-\underbrace{\lambda_{f} \mu_{h n}\left(\mu_{f o} p_{f o-h n}+\mu_{f e} p_{f e-h n}\right)}_{\text {purchases from PE funds }}, \quad \text { and } \\
& W_{f} \equiv \mu_{f o} u_{f}+\mu_{f e} u_{e}+\underbrace{\lambda_{f} \mu_{f o} \mu_{h n} p_{f o-h n}+\lambda_{f} \mu_{f e} \mu_{h n} p_{f e-h n}}_{\text {sales to corporations }}-\underbrace{\lambda_{f} \mu_{f n} \mu_{l o} p_{l o-f n}}_{\text {purchases from corporations }} \text {. }
\end{aligned}
$$

The corporate-sector welfare $W_{c}$ combines corporations' payoff flows and gains from trades. The first two terms in the definition of $W_{c}$ represent payoff flows to ho- and $l o-$ type corporations. The next term represents the inflow from selling assets to PE funds. Only lo-type corporations sell assets to PE funds with the total rate $\lambda_{f} \mu_{l o} \mu_{f n}$ and at price $p_{l o-f n}$. The last term represents the $h n$-type corporations' payments to PE funds: $p_{f o-h n}$ to $f o$-type funds with the aggregate rate of $\lambda_{f} \mu_{h n} \mu_{f o}$, or $p_{f e-h n}$ to $f e$-type funds with the aggregate rate of $\lambda_{f} \mu_{h n} \mu_{f e}$. A similar interpretation explains the financial-sector welfare $W_{f}$.

We will compare the equilibrium welfare $W$ in the presence of search frictions against two extreme situations: an autarkic economy with no functioning market or PE intermediation, 
and a centralized economy with a planner moving assets across agents without search friction. In the autarkic economy, an $n_{a}$ fraction among $n_{c}(=1)$ corporations hold assets with no trades. Thus, the steady-state welfare is $\underline{W}=n_{a}\left(n_{h} u_{h}+n_{l} u_{l}\right)$. In the centralized economy, a planner solves

$$
\begin{aligned}
& \bar{W} \equiv \max _{\mu} \mu_{h o} u_{h}+\mu_{f o} u_{f}+\mu_{f e} u_{e}+\mu_{l o} u_{l} \\
& \quad \text { subject to } \mu_{h o}+\mu_{h n}=n_{h} \text { and } \mu_{l o}+\mu_{l n}=n_{l} .
\end{aligned}
$$

The maximum welfare takes into account exogenous type changes $\rho_{u}$ and $\rho_{d}$ but ignores search frictions. It is also independent of the liquidity shock $\rho_{e}$, because assets can be transferred between $\mathrm{PE}$ funds instantaneously. The population measures $\bar{\mu}$ that achieves the maximum welfare is such that $\bar{\mu}_{h o}=\min \left\{n_{a}, n_{h}\right\}, \bar{\mu}_{f o}=\min \left\{\left(n_{a}-n_{h}\right)^{+}, n_{f}\right\}, \bar{\mu}_{l o}=$ $\left(n_{a}-n_{f}-n_{h}\right)^{+}$, and $\bar{\mu}_{i}=0$ for $i \neq h o, f o, l o$. In essence, assets are allocated to hightype corporations up to their steady-state population $n_{h}$; any remaining assets are given to $\mathrm{PE}$ funds up to $n_{f}$; and the still-remaining assets are given to low-type corporations. The maximum welfare is $\bar{W}=\bar{\mu}_{h o} u_{h}+\bar{\mu}_{f o} u_{f}+\bar{\mu}_{l o} u_{l} 17$

In Section 6, we will quantify the differences between $\underline{W} \leq W \leq \bar{W}$ using the calibrated model for the US M\&A with PE middle market.

\section{Secondary Buyouts}

In this section, we focus on SBOs which have become a common deal source and an exit channel for PE funds. We characterize their influences to the equilibrium.

SBOs Provide Liquidity and Improve Welfare. We first make simple observations: SBOs meet PE funds' demand for liquidity, so a more liquid SBO market can attenuate the

\footnotetext{
${ }^{17}$ We can characterize the maximum welfare $\bar{W}$ alternatively with a planner who is under the search friction, like agents, but can choose not to execute some transactions. The planner's problem is $W_{p}(\lambda) \equiv$ $\sup _{0 \leq \lambda_{p} \leq \lambda} \mu_{h o} u_{h}+\mu_{f o} u_{f}+\mu_{f e} u_{e}+\mu_{l o} u_{l}$, subject to $\mu$ being a solution of $P\left(n, r, u, \rho, \lambda_{p}\right)$. A large search rate allows the planner to achieve the maximum welfare approximately: i.e., $\bar{W}=\lim _{\lambda \rightarrow \infty} W_{p}(\lambda)$. An intuition will become clear after Proposition 8 the planner can slow down the corporate-M\&A market and increase the welfare to the maximum by eliminating search externalities.
} 
effects of fund liquidity shocks.

Proposition 4. While liquidity shocks reduce PE funds'values $\left(v_{f e}<v_{f o}\right)$, their influence is mitigated by more liquid SBO market $\left(\frac{\partial\left(v_{f o}-v_{f e}\right)}{\partial \lambda_{s}}<0\right)$ and vanishes when the SBO market becomes completely liquid $\left(\lim _{\lambda_{s} \rightarrow \infty}\left(v_{f o}-v_{f e}\right)=0\right)$.

More liquid SBO market improves the welfare as each SBO transaction bails a PE fund out of liquidity constraint and offers fund buyers more transaction opportunities, at no cost to any other types of agents.

Proposition 5. The welfare increases in the SBO market liquidity:

$$
\frac{\partial W}{\partial \lambda_{s}}=\frac{\partial \mu_{f o}}{\partial \lambda_{s}}\left(u_{f}-u_{e}\right)>0
$$

Intuitively, a faster SBO market allows $f e$-type PE funds to exit and re-enter as $f n$-type quickly, resulting in more $\mathrm{PE}$ funds in the investment phase $(f n)$ moving to the harvesting phase $\left(f_{o}\right)$. The welfare increases by $u_{f}-u_{e}$ for each unit measure of population shifted from $\mu_{f e}$ to $\mu_{f o}$.

While each SBO transaction benefits funds, it depletes potential trades between corporations and PE funds and may have a negative externality on corporations. For instance, the value of $f o$-type funds is higher than the value of $f e$-type funds $\left(v_{f o}>v_{f e}\right)$, so corporate buyers pay a higher price to fo-type PE funds than to $f e$-type PE funds: $p_{f o-h n}>p_{f e-h n}$. An increase of SBOs shifts some measure of $\mu_{f e}$ to $\mu_{f o}$, without affecting other types' populations. Thus, corporate buyers may pay more on purchases from PE funds (see (1)), which can reduce the corporate-sector welfare 18

Through SBOs, PE Funds Complement Each Other. Although PE funds compete for intermediation opportunities, self-interested PE funds help each other out through SBOs. When there is a greater number of PE funds in the market, exiting funds under pressure can sell more quickly. At the same time, PE funds looking to buy assets benefit from immediately available opportunities from exiting funds. The mutual benefits of SBOs among PE funds can be so large as to dominate the reduction in value from narrower buy-sell spreads due

\footnotetext{
${ }^{18} \mathrm{~A}$ formal proof would be complicated as transaction prices depend on $\lambda_{s}$.
} 
to the increased competition. Increases in SBO liquidity, i.e., higher $\lambda_{s}$, raises the marginal benefit $\frac{\partial v_{f n}}{\partial n_{f}}$ of having additional funds in the market:

Proposition 6. There exists $\bar{\lambda}_{s}>0$ and a function $\bar{n}_{f}\left(\lambda_{s}\right)$ such that, if the SBO market is liquid enough $\left(\lambda_{s}>\bar{\lambda}_{s}\right)$ and there are not too many PE funds $\left(n_{f}<\bar{n}_{f}\left(\lambda_{s}\right)\right)$, then

1. PE funds' value increases in the number of PE funds $\left(\frac{\partial v_{f n}}{\partial n_{f}}>0\right)$, and;

2. There is a complementarity between the SBO-market liquidity and the number of PE funds $\left(\frac{\partial^{2} v_{f n}}{\partial \lambda_{s} \partial n_{f}}>0\right)$.

The proof of Proposition 6 in Subsection B.3 obtains the closed-form expression of the complementarity $\left(\frac{\partial^{2} v_{f n}}{\partial \lambda_{s} \partial n_{f}}\right)$ at $n_{f} \approx 0$. The complementarity is strong if there is an oversupply of assets relative to the number of corporate buyers; for otherwise, exiting funds could easily sell assets through the PBO market, and the benefits of SBOs would be insignificant. Moreover, the complementarity is stronger if the liquidity shocks are more severe (in intensity, $u_{f}-u_{e}$ or frequency, $\left.\rho_{e}\right)$.

When the number of $\mathrm{PE}$ funds increases, competition amongst $\mathrm{PE}$ funds in chasing intermediation opportunities becomes stiffer. The value reduction due to competition may ultimately cancel out the benefit from complementarities. Figure 2 shows how the value of $f n$-type fund (i.e., a direct competitor of a new entrant) responds to $n_{f}$ and $\lambda_{s}$, with other parameters being fixed at the value calibrated in Section 6 . The vertical line at $n_{f}=n_{f}^{*}$ and the curve for $\lambda_{s}=\lambda_{s}^{*}$ correspond to the observed number of PE funds and the calibrated search rate for SBOs 19 The figure confirms Proposition 6. At the calibrated level of market parameters, the benefit from complementarity among PE funds due to SBOs supersedes competitive pressures. However, for large $n_{f}$, lower spreads due to increased competition for intermediation opportunities turn out to be the dominant force impacting fund valuation.

Our calibration analysis in Section 6 also shows that SBO exits play an increasingly important role in the growth in private equity. As we increase the number of PE funds, holding other parameters at calibrated values, we find that an increasing fraction of funds exit through SBOs rather than PBOs (Figure 3). While it is obvious that the number of

\footnotetext{
${ }^{19}$ The calibrated search rates $\lambda=\left(\lambda_{c}, \lambda_{f}, \lambda_{s}\right)$ tend to be very large due to our normalization of $n_{c}=1$; see Section 5 for the discussions. This large $\lambda$ motivates us to study a fast-search market.
} 


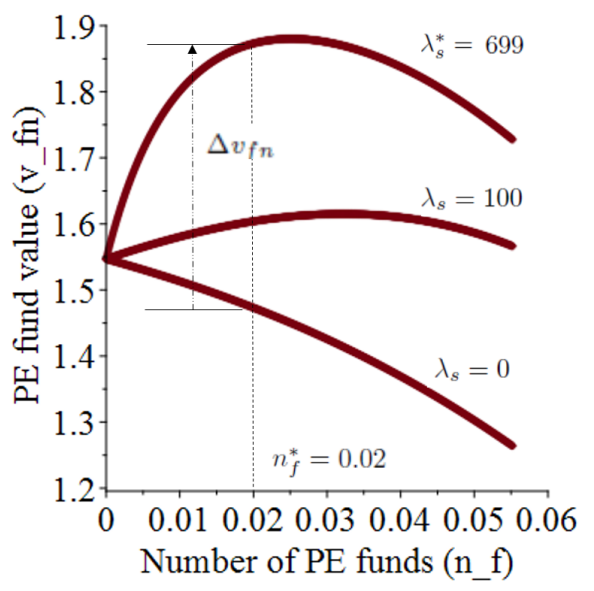

Figure 2: The y-axis shows $v_{f n}$ for various values of $n_{f}$ at calibrated values of all other market parameters (see Section 6). The topmost curve is for the calibrated value of $\lambda_{s}$ while the bottom two curves are based on the counter-factual parameter values. The observed number of funds is $n_{f}^{*}=0.02$ (normalized by the number of corporations). The increase in fund valuation $\Delta v_{f n}$ represents the contribution by SBOs, i.e., an increase of $\lambda_{s}$ from 0 to the calibrated value $\lambda_{s}^{*}=699$.

SBOs increase with the growth in PE funds, our model explains a tandem increase in the share of exits through SBOs. This prediction of the model is consistent with a conspicuous change in the private equity market that has been extensively documented (see Section 6 for details).

\section{$5 \quad$ Fast-search Markets}

We consider a steady-state equilibrium with high search rates (i.e., a large $\left.\lambda=\left(\lambda_{c}, \lambda_{f}, \lambda_{s}\right)\right)$ and find a closed-form expression for the equilibrium. This characterization highlights several important equilibrium properties, such as asymptotic inefficiency and search externalities.

Our calibration exercise in Section 6 also motivates us to study a fast-search market. As we normalize the number of corporations to $n_{c}=1$, we proportionally re-scale the number of PE funds $n_{f}$ and the number of transactions in each market. Let $N_{c}$ be the total number of corporations, with $N_{i}$ for $i \in \mathcal{T}_{c}$ being the number corporations of each type $i$. If each pair 


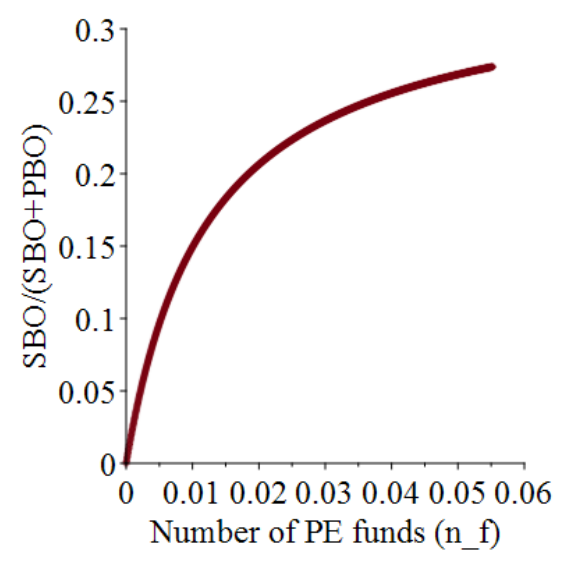

Figure 3: The y-axis shows the fraction of SBOs among all exits by $\mathrm{PE}$ funds $\left(\frac{\mathrm{SBO}}{\mathrm{SBO}+\mathrm{PBO}}=\frac{\lambda_{s} \mu_{f e} \mu_{f n}}{\lambda_{f} \mu_{f e} \mu_{h n}+\lambda_{s} \mu_{f e} \mu_{f n}}\right)$ for various values of $n_{f}$ at calibrated values of all other market parameters (see Section 6).

of corporations meet at a Poisson rate $\Lambda_{c}$, the total number of corporate-M\&A deals (say, per year), with normalization, would be

$$
\Lambda_{c} N_{l o} N_{h n} / N_{c}=\left(\Lambda_{c} N_{c}\right)\left(N_{l o} / N_{c}\right)\left(N_{h n} / N_{c}\right)=\left(\Lambda_{c} N_{c}\right) \mu_{l o} \mu_{h n}
$$

The calibrated search rate $\lambda_{c}=\Lambda_{c} N_{c}$ tends to be much higher than other parameters.

We set up a formal fast-search market as follows. Given any exogenous parameters $\theta \equiv(n, r, u, \rho, \lambda)$, we increase meeting rates $\left(\lambda_{c}, \lambda_{f}, \lambda_{s}\right)$, while preserving the relative ratios. That is, we consider a sequence of markets $\theta^{\kappa} \equiv(n, r, u, \rho, \kappa \lambda)$, where $\kappa \lambda=\left(\kappa \lambda_{c}, \kappa \lambda_{f}, \kappa \lambda_{s}\right)$. We analyze the steady-state solution $\left(\mu^{\kappa}, v^{\kappa}\right)$ in the limit as $\kappa$ increases to infinity. To ease expositions, we assume a regular environment: $n_{a} \notin\left\{n_{h}, n_{h}+n_{f}\right\}$.

The fast-search market is well-defined as the steady-state population measures $\mu^{\kappa}$ converge (the convergence of $v^{\kappa}$, as a linear function of $\mu^{\kappa}$, follows immediately (see (14)). The speed of convergence is $O(1 / \kappa)$ which gives a precise sense of how closely a fast-search equilibrium would approximate an equilibrium of the calibrated market (in our calibration, $\kappa \approx 103,000):$

Proposition 7. (Convergence and Convergence Speed) Take any regular environment $\theta$. 


\begin{tabular}{lccc}
\hline \hline & A. $n_{a}<n_{h}$ & B. $n_{h}<n_{a}<n_{h}+n_{f}$ & C. $n_{h}+n_{f}<n_{a}$ \\
\hline $\bar{\mu}_{h o}=$ & $n_{a}$ & $n_{h}$ & $n_{h}$ \\
$\bar{\mu}_{f o}=$ & 0 & $n_{a}-n_{h}$ & $n_{f}$ \\
$\bar{\mu}_{l o}=$ & 0 & 0 & $n_{a}-n_{f}-n_{h}$ \\
$\bar{\mu}_{f e}=$ & 0 & 0 & 0 \\
\hline$\mu_{h o}^{*}=$ & $n_{a}$ & $n_{h}$ & $n_{h}$ \\
$\mu_{f o}^{*}=$ & 0 & $n_{a}-n_{h}$ & $<n_{f}$ \\
$\mu_{l o}^{*}=$ & 0 & 0 & $n_{a}-n_{f}-n_{h}$ \\
$\mu_{f e}^{*}=$ & 0 & 0 & $>0$ \\
\hline
\end{tabular}

Table 1: The population under fast search $\left(\mu^{*}\right)$ and the efficient allocation $(\bar{\mu})$.

For any $i \in \mathcal{T}$, the population limit $\mu_{i}^{*} \equiv \lim _{\kappa \rightarrow \infty} \mu_{i}^{\kappa}$ and the convergence speed $\mu_{i}^{* *} \equiv$ $\lim _{\kappa \rightarrow \infty} \kappa\left(\mu_{i}^{\kappa}-\mu_{i}^{*}\right)$ exist.

The appendix has the closed-form expressions for the limit populations and the convergence speed.

\subsection{Inefficiency and Search Externality}

We study welfare in the fast-search market. The closed-form expression of the unique steadystate population allows us to compare the welfare in this market with the maximum conceivable welfare $\bar{W}$, which is induced by the population measure $\bar{\mu}$ (see the upper part of Table 1) that we obtained in Subsection 3.3. We compare the efficient allocation $\bar{\mu}$ with the fast-search population $\mu^{*}$ (the lower part of Table 1) ${ }^{20}$

The welfare of the fast-search market is defined by $W^{*} \equiv W\left(\mu^{*}\right)$, where $\mu^{*}=\lim _{\kappa \rightarrow \infty} \mu^{\kappa}{ }^{21}$ We find that with a sufficiently large number of assets $\left(n_{a}>n_{h}+n_{f}\right)$, which is indeed the case of our calibration in Section 6 , the fast-search equilibrium does not allocate assets efficiently:

Proposition 8. (Fast-search Market: Welfare) As $\kappa \rightarrow \infty$,

A. If $n_{a}<n_{h}$, then $W^{*}=\bar{W}$, which is independent of $u_{f}, u_{e}, \lambda_{s}$, and $\lambda_{c}$.

\footnotetext{
${ }^{20}$ The fast-search market population $\mu^{*}$ is constructed from Lemma 4 and Lemma 5 in Subsection C.1. In Case $\mathrm{C}, \mu_{f e}^{*}=\frac{n_{f}}{1+\frac{\rho_{u} \mu_{l n}^{*}}{\rho_{e} \mu_{l_{o}}^{*}} \frac{\lambda_{f o}^{*}+\lambda_{s} n_{f}}{\lambda_{c} \mu_{l_{o}}^{*}+\lambda_{f} n_{f}}}$ and $\mu_{f o}^{*}=n_{f}-\mu_{f e}^{*}$.

${ }^{21} W\left(\mu^{\kappa}\right)$ converges at the same speed $O(1 / \kappa)$ as $\mu^{\kappa}$, because the welfare $W\left(\mu^{\kappa}\right)$ is a linear aggregation of the population measures $\left(\mu_{i}^{\kappa}\right)_{i \in \mathcal{T}}$.
} 
B. If $n_{h}<n_{a}<n_{h}+n_{f}$, then $W^{*}=\bar{W}$, which is strictly increasing in $u_{f}$ and independent of $u_{e}, \lambda_{s}$, and $\lambda_{c}$.

C. If $n_{h}+n_{f}<n_{a}$, then $W^{*}$ is strictly less than $\bar{W}$, strictly increasing in $u_{f}, u_{e}$, and $\lambda_{s}$, and strictly decreasing in $\lambda_{c}$.

The characterization of the fast-search equilibrium welfare depends on the number of assets $\left(n_{a}\right)$ relative to the number of potential buyers $\left(n_{h}, n_{f}\right)$.

Suppose that the fast-search market has sufficiently many potential buyers $\left(n_{a}<n_{h}+n_{f}\right)$ as in Cases A and B (the first two columns in Table 1). Fast search allows corporations and funds to quickly transfer assets from low-type corporations $(l o)$ and exiting funds $(f e)$ to high-type corporations $(h n)$ and, in Case B, also to funds at the investment phase $(f n)$. Accordingly, the steady-state population $\mu^{*}$ equals the efficient allocation $\bar{\mu}$ and achieves the maximum welfare $\left(W^{*}=\bar{W}\right)$. The comparative static of the welfare becomes trivial: the maximum welfare is dependent on payoff flows (e.g., $u_{f}$ ) only if the corresponding type's population (resp., $\mu_{f o}$ ) is non-zero. In either case, the impact of a liquidity shock is zero. PE funds transfer assets without holding any inventory (Case A) just like, e.g., in Rubinstein and Wolinsky (1987); or, they hold assets, but funds under liquidity constraints transfer assets to others through speedy SBOs (Case B), as is the case in Duffie, Gârleanu, and Pedersen (2005).

The more interesting case is when there is a surplus of tradable assets relative to potential buyers $\left(n_{h}+n_{f}<n_{a}\right)$, as in Case C (the third column in Table 1). Since corporate or PE buyers can quickly find sellers and purchase assets, there is negligible left-over supply. Hence, a significant fraction of exiting funds $(f e)$ will find it difficult to offload their assets. The welfare loss is $\bar{W}-W^{*}=\mu_{f e}^{*}\left(u_{f}-u_{e}\right)>0$. We could slow down the corporate M\&A market, maintain more corporate buyers in steady-state and improve welfare $\left(\frac{\partial W^{*}}{\partial \lambda_{c}}<0\right)$. Then, PE funds under liquidity constraints sell assets more quickly. In this sense, each corporate-M\&A deal creates search externalities to PE funds. 


\subsection{M\&A Deal Volumes}

Corporations, PE funds and M\&A practitioners are keenly focused on the current and future levels of activity in M\&A markets. In this section, we study how the transaction volumes are determined by exogenous parameters, such as search frictions and transition rates.

As before, we consider $\theta^{\kappa}=(n, r, u, \rho, \kappa \lambda)$ with $\kappa \rightarrow \infty$. For each submarket $m \in \mathcal{M}$, with a seller's type $s$ and the buyer's type $b$, the trade volume is

$$
\eta_{m}^{\kappa} \equiv\left(\kappa \lambda_{m}\right) \mu_{s}^{\kappa} \mu_{b}^{\kappa}
$$

We characterize the limit trade volumes:

Proposition 9. (Fast-search Market: Trade Volumes) For each submarket $m \in \mathcal{M}$, trade volume in the limit $\eta_{m}^{*} \equiv \lim _{\kappa \rightarrow \infty} \eta_{m}^{\kappa}$ is given by:

\begin{tabular}{lccc}
\hline \hline & $A . n_{a}<n_{h}$ & $B . n_{h}<n_{a}<n_{h}+n_{f}$ & $C . n_{h}+n_{f}<n_{a}$ \\
\hline$\eta_{l o-h n}^{*}=$ & $\lambda_{c} \mu_{l o}^{* *} \mu_{h n}^{*}$ & 0 & $\lambda_{c} \mu_{l o}^{*} \mu_{h n}^{* *}$ \\
$\eta_{l o-f n}^{*}=$ & $\lambda_{f} \mu_{l o}^{* *} \mu_{f n}^{*}$ & $\lambda_{f} \mu_{l o}^{* *} \mu_{f n}^{*}$ & $\lambda_{f} \mu_{l o}^{*} \mu_{f n}^{* *}$ \\
$\eta_{f o-h n}^{*}=$ & $\lambda_{f} \mu_{f o}^{* *} \mu_{h n}^{*}$ & $\lambda_{f} \mu_{f o}^{*} \mu_{h n}^{* *}$ & $\lambda_{f} \mu_{f o}^{*} \mu_{h n}^{* *}$ \\
$\eta_{f e-h n}^{*}=$ & 0 & 0 & $\lambda_{f} \mu_{f e}^{*} \mu_{h n}^{* *}$ \\
$\eta_{f e-f n}^{*}=$ & 0 & $\lambda_{s} \mu_{f e}^{* *} \mu_{f n}^{*}$ & $\lambda_{s} \mu_{f e}^{*} \mu_{f n}^{* *}$ \\
\hline
\end{tabular}

where (i) $\mu^{*} \equiv \lim _{\kappa \rightarrow \infty} \mu^{\kappa}$ is the population limit, and (ii) for type $i$ with $\mu_{i}^{*}=0, \mu_{i}^{* *} \equiv$ $\lim _{\kappa \rightarrow \infty} \kappa \mu_{i}^{\kappa}$ is the convergence speed ${ }^{22}$

With a large number of high-type corporations (Case A), SBOs are unnecessary for PE funds; if the deficit of high-type corporations is supplemented by PE funds (Case B), corporate sellers resort to the $P B O$ market and there are no corporate-MEA deals; with an excess supply of tradable assets (Case $C$ ), there are transactions in all submarkets.

The trade volumes under fast search follow from the convergence and the convergence speed of population measures (Proposition 7). For each submarket $m \in \mathcal{M}$, because of fast search, the measure of either buyers or sellers vanishes: i.e., $\mu_{b}^{*}=0$ or $\mu_{s}^{*}=0$. If $\mu_{s}^{*}=0$, then $\eta_{m}^{\kappa}=\left(\kappa \lambda_{m}\right) \mu_{b}^{\kappa} \mu_{s}^{\kappa}=\lambda_{m} \mu_{b}^{\kappa}\left(\kappa \mu_{s}^{\kappa}\right) \rightarrow \lambda_{m} \mu_{b}^{*} \mu_{s}^{* *}$ as $\kappa \rightarrow \infty$.

\footnotetext{
${ }^{22}$ See Lemma 5 in Subsection C.1 and Section S.1 in Supplemental Appendix for the closed-form expressions.
} 
Proposition 9 suggests that all submarkets are active under fast search only if the market has an excess supply of tradable assets $\left(n_{a}>n_{h}+n_{f}\right)$. In a calibration of our model (Section 6), we observe non-negligible transaction volumes in all submarkets, which suggests Case $\mathrm{C}$ of the fast-search market best approximates the US M\&A with PE middle market.

The trade volumes also identify the main drivers of the convergences of certain population measures. For example, $\mu_{f o}^{*}=0$ in Case A could be due to the fact that (i) funds do not purchase assets due to a vanishingly small number of corporate sellers $(l o)$, or (ii) funds do acquire assets, but quickly re-sell to corporate buyers $(h n)$. According to Proposition 9, PE funds buy and sell a significant number of assets even in fast-search markets, like middlemen in Rubinstein and Wolinsky (1987), and there are no SBO transactions. A large number of SBO transactions observed in the data thus seems to rule out this case as an empirically plausible one. Similarly, the vanishing corporate sellers $(l o)$ and buyers $(h n)$ in Case B is the result of an efficient PBO market rather than the corporate-M\&A market - the number of corporate-M\&As $\left(\eta_{l o-h n}^{*}\right)$ is indeed vanishingly small.

We conclude this section with a comparative static analysis for trade volumes relative to search frictions and transition rates, as summarized in Table $2{ }^{23}$

\begin{tabular}{cccccc}
\hline \hline & $\lambda_{c}$ & $\lambda_{f}$ & $\lambda_{s}$ & $\left(\rho_{u}, \rho_{d}\right)_{(\text {with a fixed ratio })}$ & $\rho_{e}$ \\
\hline$\eta_{l o-h n}^{*}$ & + & - & 0 & + & 0 \\
$\eta_{l o-f n}^{*}$ & - & + & 0 & + & 0 \\
$\eta_{f o-h n}^{*}$ & - & + & + & + & - \\
$\eta_{f e-h n}^{*}$ & - & + & - & + & + \\
$\eta_{f e-f n}^{*}$ & - & - & + & + & + \\
\hline
\end{tabular}

Table 2: Comparative statics of trade volumes. Each $+($ or, -$)$ indicates that the corresponding volume are non-decreasing (resp., non-increasing) in the parameter, and 0 indicates that the volume is independent of the parameter.

Most results are intuitive. If corporations' types are more volatile (i.e., larger $\rho_{u}$ and $\rho_{d}$ with a fixed ratio), assets will be transferred across agents frequently, ultimately from low-

\footnotetext{
${ }^{23}$ The proofs are tedious and follow directly from $\mu^{*}$ and $\mu^{* *}$ in Table 1 and Section S.1 of Supplemental Appendix, so we omit. As an example, take $\eta_{l o-h n}^{*}$ and $\lambda_{c}$. Note that $\eta_{l o-h n}^{*}=\lambda_{c} \mu_{l o}^{*} \mu_{h n}^{* *}$. In Cases A and B, $\mu_{l o}^{*}=0$, so $\eta_{l o-h n}^{*}$ is independent of $\lambda_{s}$. In Case C, $\mu_{h n}^{* *}=\frac{\rho_{u} \mu_{l n}^{*}}{\lambda_{c} \mu_{l o}^{*}+\lambda_{f}\left(\mu_{f o}^{*}+\mu_{f e}^{*}\right)}=\frac{\rho_{u} \mu_{l n}^{*}}{\lambda_{c} \mu_{l o}^{*}+\lambda_{f} n_{f}}$. Note that $\mu_{l o}^{*}$ and $\mu_{l n}^{*}$ are independent of $\lambda_{c}$. Thus, the volume $\eta_{l o-h n}^{*}$ is increasing in $\lambda_{c}$ because $\frac{\partial}{\partial \lambda_{c}}\left(\frac{\lambda_{c}}{\lambda_{c} \mu_{l o}^{*}+\lambda_{f} n_{f}}\right)=\lambda_{f} n_{f}>0$.
} 
type corporations to high-type corporations, with possible PE intermediations. Fast search among corporations (i.e., higher $\lambda_{c}$ ) allows them to transact directly (i.e., higher $\eta_{l o-h n}^{*}$ ), resulting in fewer intermediation opportunities for PE funds. The parameters for the SBO market $\left(\lambda_{s}\right.$ and $\left.\rho_{e}\right)$ only shift the population measures between $f o$ and $f e$. Therefore, these parameters do not affect the trade volume in the corporate-M\&A market $\left(\eta_{l o-h n}^{*}\right)$ and only shift volumes between two kinds of trades in the PBO market: $\eta_{f o-h n}^{*}$ and $\eta_{f e-h n}^{*}$.

The positive response of $\eta_{f e-h n}^{*}$ to $\lambda_{f}$ is somewhat surprising. Fast search in the PBO market (i.e., higher $\kappa \lambda_{f}$ ) orchestrates more transactions between exiting funds $(f e)$ and corporate buyers $(h n)$. However, as funds are able to sell assets before receiving liquidity shocks, fewer funds enter the exit phase, which could potentially reduce the trade volume between exiting funds and corporate buyers. It turns out that the former effect of $\lambda_{f}$ dominates the latter.

\section{Calibration}

We perform a calibration exercise to determine how well our model can fit the vital statistics of the M\&A with PE market. We ask various quantitative questions such as: (i) Is there an over or undersupply of tradable assets relative to the number of corporate buyers and PE funds? (ii) How are fund valuations affected by liquidity provision through SBOs and fund managers' operational improvement? (iii) What is the impact of PE entry on fund valuation and transaction prices? (iv) What are the welfare losses associated with search frictions?

Our model has 14 exogenous parameters: $n=\left(n_{c}, n_{f}, n_{a}\right), u=\left(u_{l}, u_{h}, u_{f}, u_{e}\right), \rho=$ $\left(\rho_{u}, \rho_{d}, \rho_{e}\right), \lambda=\left(\lambda_{c}, \lambda_{f}, \lambda_{s}\right)$ and $r$. Seven parameters $(n, u)$ are either directly observed or obtained from other empirical studies, and the remaining seven parameters $(\rho, \lambda, r)$ are estimated to fit the model statistics in Table 3 to the data. The calibrated parameters are reported in Table 4.

Data and Methodology We focus on the U.S. M\&A with PE middle market. For corporations, we consider companies with annual revenues between $\$ 20$ million and $\$ 1,000$ million. We exclude small companies, for which there is no reliable data on M\&A and PE activities, and large companies, which are often listed on stock exchanges and transact at different price 
multiples. The focus on the mid-size companies is to maintain an overall homogeneity in our sample set. According to the latest available U.S. Economic Census Data (2012), there are approximately 102,626 mid-size companies. We normalize $n_{c}=1$ and apply the same rescaling to the number of $\mathrm{PE}$ funds, assets, trading volumes, etc.

The primary data is from the 2018 US PE middle market report and the M\&A report by Pitchbook Data Inc. The data includes deals with transaction values between $\$ 25$ million and $\$ 1,000$ million. From this dataset we estimate that there are 1,893 PE funds targeting the middle market ( $n_{f} \simeq 0.02$ with normalization) ${ }^{24}$ The data set also provides the number of transactions for each submarket, as summarized in Table 3 along with the model statistics. The total number of M\&A middle market deals was an average of 9,626 per year from 2007 to 2017. In the same period, PE funds acquired an average of 1,799 mid-size firms per year, of which $359(\approx 20 \%)$ are through SBOs and 1,440 are through PBOs. The steady state of our model implies that the number of buyouts must be equal to the number of exits (i.e, $\eta_{f e-h n}+\eta_{f o-h n}=\eta_{l o-f n}$ ). The total number of M\&A deals is then equal to the sum $\eta_{l o-h n}+2 \eta_{l o-f n}+\eta_{f e-f n}$, which gives us an estimate of 6,387 corporate M\&A deals per year. The transaction volumes such as $\eta_{l o-h n}=\lambda_{c} \mu_{l o} \mu_{h n}$ allow us to identify the search intensities $\lambda \sqrt{25}$

For the average time to sell for corporations and PE funds, we resort to various reports available online (see Table S.1 in Supplemental Appendix) and take their average ${ }^{26}$ For the average time to sell, we include both time taken in the preparation process and the listing-to-sale process ${ }^{27}$ We estimate, mainly from reports prepared by selling agents such

\footnotetext{
${ }^{24}$ Pitchbook reports the number of PE funds raised in each year targeting the middle market. We cumulate these numbers for 2007-2018, as the average lifespan of PE funds is 12 years (Metrick and Yasuda (2010)).

${ }^{25}$ As explained in Section 5 due to our normalization $n_{c}=1$, the calibrated search rate $\lambda_{c}$ (and similarly for $\lambda_{f}$ and $\lambda_{s}$ ) should be interpreted carefully. Let $N_{c}$ be the total number of companies in our data, and $N_{l o}$ and $N_{h n}$ be the numbers for the corresponding types. If the search rate in the corporate-M\&A market is $\Lambda_{c}$, then the volume of corporate-M\&As, after normalization, is $\Lambda_{c} N_{l o} N_{h n} / N_{c}=\left(\Lambda_{c} N_{c}\right)\left(N_{l o} / N_{c}\right)\left(N_{h n} / N_{c}\right)=$ $\left(\Lambda_{c} N_{c}\right) \mu_{l o} \mu_{h n}$. The calibrated rate represents $\Lambda_{c} N_{c}$.

${ }^{26}$ We are unaware of any research paper that estimates the average selling time in the M\&A market. For public markets, Boone and Mulherin (2007) use the SEC filings and estimate corporate sellers' average selling time from the private initiation to the deal completion.

${ }^{27} \mathrm{~A}$ seller of a firm needs to prepare a Confidential Information Memorandum (CIM) in conjunction with the seller's agent: an investment banker, a M\&A broker, or a business broker. The seller also prepares requisite documents for subsequent steps, including due diligence. Once the firm is ready, the seller approaches prospective buyers. Upon finding buyers with serious interest, the firm enters a negotiation process followed
} 


\begin{tabular}{lcc}
\hline \hline Description & Data & $\begin{array}{c}\text { Model Statistic } \\
\text { (for normalized values) }\end{array}$ \\
\hline Corporate M\&As & 6387 & $\eta_{l o-h n}=\lambda_{c} \mu_{l o} \mu_{h n}$ \\
Primary Buyouts (PBOs) & 1440 & $\eta_{l o-f n}=\lambda_{f} \mu_{l_{o}} \mu_{f n}$ \\
Secondary Buyouts (SBOs) & 359 & $\eta_{f n-f e}=\lambda_{s} \mu_{f n} \mu_{f e}$ \\
Avg time to sell for corporations & 1.25 years & $E\left[\tau_{s c}\right]($ eq. $(2))$ \\
Avg time to sell for PE funds & 0.91 years & $E\left[\tau_{s f}\right]$ (eq. (3)) \\
Fund performance (PME) & 1.01 & PME (eq. (4T)) \\
Price multiple (EV/EBITDA) & 9.0 & $P_{l o-h n} / u_{l}$ \\
\hline
\end{tabular}

Table 3: Key Statistics on M\&A and Private Equity

as business brokers or investment bankers, that the preparation process for PE funds takes only an average of 2 months - much shorter than an average of 6 months for the corporate sector. Portfolio firms of PE funds are usually in a better state of readiness to approach the market due to high-quality governance, accounting, and information systems. The listing-tosale process takes an average of 9 months for selling agents. In total, selling a firm takes an average of 11 months (0.91 years) for PE funds and 15 months (1.25 years) for corporations.

The statistics above corresponds to the model statistics $E\left[\tau_{s c}\right]$ and $E\left[\tau_{s f}\right]$ : the average time to sell for corporations and PE funds. Each seller-buyer meeting arrives according to a Poisson process, so the time until the first meeting by a corporate seller follows an exponential distribution with parameter $\lambda_{c} \mu_{h n}+\lambda_{f} \mu_{f n}$. Thus, the expected time to sell is

$$
E\left[\tau_{s c}\right]=\frac{1}{\lambda_{c} \mu_{h n}+\lambda_{f} \mu_{f n}}
$$

A similar, but more involved, calculation (see Supplemental Appendix) gives the expected time to sell for PE funds:

$$
E\left[\tau_{s f}\right]=\frac{1}{\lambda_{f} \mu_{h n}+\rho_{e}}+\frac{\rho_{e}}{\lambda_{f} \mu_{h n}+\rho_{e}}\left(\frac{1}{\lambda_{f} \mu_{h n}+\lambda_{s} \mu_{f n}}\right) .
$$

A commonly used performance measure of PE funds is the Public Market Equivalent (PME) introduced by Kaplan and Schoar (2005) (see also Sorensen and Jagannathan (2015)). by a due-diligence process. 
Formally, PME is defined as

$$
P M E=\frac{\text { Present value of distributions to fund investors }}{\text { Present value of capital calls made by fund investors }} .
$$

PME is the ratio of cash outflows to investors and cash contributions by investors, both discounted at the public market total return (e.g., S\&P 500 index) after subtracting management fees paid to the fund managers - see Supplemental Appendix for a derivation of the closed-form expression 28 A PME of one is indicative of a fund's performance in line with the public market, and a lower PME indicates underperformance.

We take the average PME of 1.01 from various estimates. Kaplan and Schoar (2005) estimate an average PME of 0.93 for PE funds in the period 1980-1994, while Phalippou and Gottschalg (2008), using similar dataset but different methodology, report an average PME of 0.88. Harris, Jenkinson, and Kaplan (2014), on the other hand, report significantly better performance with an average PME of 1.22 for the period 1984-2008. The estimates of PME by PitchBook Data, Inc. yields an average of 1.00 for the period 2006-15.

The most widely used metric for the relative prices of M\&A deals is the EV/EBITDA multiple. The transaction multiple for corporate-M\&A deals, which is matched to our model's $P_{l o-h n} / u_{l}$, is on average 9.0 from 2005 to 201729

We now turn to the payoff parameters. We normalize the low-type corporations' flow payoff $u_{l}=1$. For the flow payoff $u_{f}$, we use Muscarella and Vetsuypens (1990), Opler (1992) and Andrade and Kaplan (1998). Each of these papers estimate the increase in operating profits (or cash flows) for firms after fund buyouts as 23.5\%, 16.5\%, and $52.9 \%$, respectively. This increase is mainly due to improved incentive schemes for firm managers and better governance. We take the average and set $u_{f}=1.3$ (i.e., $30 \%$ increase from $u_{l}$ ), which is also close to the average $28.5 \%$ of the takeover premium paid by PE acquirers (Bargeron, Schlingemann, Stulz, and Zutter, 2008). For the flow payoff net of liquidity cost $u_{e}$, Nadauld, Sensoy, Vorkink, and Weisbach (2016) find that investors under liquidity shocks sell their ownership in PE funds to other investors at a $13.8 \%$ discount. This observation motivates

\footnotetext{
${ }^{28}$ According to Metrick and Yasuda (2010), the management fees are usually $2 \%$ of the committed capital and paid from the inception of the fund until its liquidation.

${ }^{29}$ See a recent report on EV/EBITDA by FactSet Research Systems Inc. at https://www.factset.com/ hubfs/mergerstat_em/monthly/US-Flashwire-Monthly.pdf.
} 


\begin{tabular}{llcc}
\hline \hline & Parameters & Variable & Value (normalized/data) \\
\hline & No. of Corporations & $n_{c}$ & $1.0(102,626)$ \\
& No. of PE funds & $n_{f}$ & $0.02(1,893)$ \\
No. of Assets & $n_{a}$ & 0.5 \\
(Observed) & Flow Payoff low type & $u_{l}$ & 1 \\
& Flow Payoff high type & $u_{h}$ & 1.4 \\
& Flow Payoff PE (harvesting) & $u_{f}$ & 1.3 \\
& Flow Payoff PE (exiting) & $u_{e}$ & 1.1 \\
\hline Low valuation shock & $\rho_{d}$ & 0.23 \\
& High valuation shock & $\rho_{u}$ & 0.16 \\
& Liquidity shock & $\rho_{e}$ & 0.38 \\
Match intensity (corporations) & $\lambda_{c}$ & 46.1 \\
& Match intensity (PE to corp) & $\lambda_{f}$ & 61.7 \\
Match intensity (PE to PE) & $\lambda_{s}$ & 699 \\
Discount rate & $r$ & $11.8 \%$ \\
\hline
\end{tabular}

Table 4: Fitted Parameters of Calibration

our choice of $u_{e}=(1-0.138) \times u_{f} \simeq 1.1$.

It is difficult to observe the payoff improvement from acquisitions, $u_{h}-u_{l}$, because the target's and acquirer's operations often blend together seamlessly. As such, we seek indirect evidence from the premium paid by corporate buyers. Betton, Eckbo, and Thorburn (2008) reports an average $43 \%$ takeover premium over 4,880 acquisitions during 1980-2002, and Bargeron, Schlingemann, Stulz, and Zutter (2008) find that the takeover premium paid by a private acquirer is $40.9 \%$. From these estimates, we choose $u_{h}=1.4$.

We do not directly observe the number of tradable assets. In our benchmark analysis, we set the number of tradable assets at half of the overall mass of corporations, i.e. $n_{a}=0.5$. We also estimated the parameter values in Table 4 with $n_{a}=0.25$ and $n_{a}=0.75$, and found insignificant changes to the estimates, except for $\rho_{d}$ and $\rho_{u}$. Finally, our choice for the bargaining power of 0.5 is consistent with an equal split of the dollar gain between acquirers and target found in Ahern (2012).

We estimate the remaining seven parameters $\beta \equiv(\rho, \lambda, r)$ that best explain the key statistics in Table 3. Each choice of the remaining parameters' values, together with the directly observed parameters $(n, u)$, defines an M\&A with PE market $\theta=(n, r, u, \rho, \lambda)$. We 
compute the statistics $Y_{i}(\beta ; n, u)$ for each row $i=1, \ldots, 7$ in Table 3 and compare them with the observed data $Y_{i}^{\text {obs }}$. We estimate the remaining parameters $\beta$ by minimizing the sum of squared residuals (SSR), subject to positive trade gains in the unique steady-state solution of the market $(\beta, n, u)$ :

$$
\begin{aligned}
& \min _{\beta} S S R(\beta ; n, u) \equiv \sum_{i=1}^{7}\left(\frac{Y_{i}(\beta ; n, u)-Y_{i}^{\text {obs }}}{Y_{i}^{\text {obs }}}\right)^{2} \\
& \text { subject to } \quad g_{m}(\beta ; n, u) \geq 0, \quad \text { for each } m \in \mathcal{M} .
\end{aligned}
$$

The estimated parameter values are summarized in the lower section of Table 4. Despite its stylized nature, our model fits the observed data with a high degree of accuracy: the minimum SSR is approximately $2.9 \times 10^{-5}$.

The estimated parameters are of reasonable magnitudes. The estimated corporate transition rates $\rho_{u}$ and $\rho_{d}$ suggest that the type transitions (from high to low, and vice versa) happen within 6.3 to 4.3 years on average. The meeting rates $\lambda=\left(\lambda_{c}, \lambda_{f}, \lambda_{s}\right)$ are of a much higher order, due to normalization, as discussed in Section 5. The meeting rate between corporations and PE funds is higher than between corporations $\left(\lambda_{f}>\lambda_{c}\right)$. The calibrated inter-PE meeting rate $\lambda_{s}$ is much higher than the other two, reflecting the high volume of SBOs relative to the small fraction of PE funds. Lastly, the estimated discount rate $r=11.8 \%$, although high, seems reasonable given that the assets traded are mostly mid-size private companies 30

Results The calibration results in Table 5 answer the quantitative questions (i)-(iv) at the beginning of this section.

First, there is an oversupply of tradable assets. The calibrated $\rho_{u}$ and $\rho_{d}$ suggest $n_{h} \equiv$ $\frac{\rho_{u}}{\rho_{u}+\rho_{d}}=0.40$, implying an overall shortage of corporate buyers and fund buyers relative to

\footnotetext{
${ }^{30} \mathrm{~A}$ large literature reports that the expected rate of return is often higher for illiquid assets such as mid-size private firms (Amihud, Mendelson, and Pedersen, 2006). Another example is restricted shares issued by publicly traded companies in private placements, which are identical to the public stocks, except for restrictions in trading; they are priced about $20 \%$ to $45 \%$ lower than the public stock prices (Silber, 1991). Regarding transaction multiples, Koeplin, Sarin, and Shapiro (2000) report that private companies are acquired at 20-30\% discount, relative to similar public companies. Regarding risk-return studies for $\mathrm{PE} / \mathrm{VC}$ funds, Cochrane (2005) estimates the rate of return in a CAPM-based approach at $15 \%$.
} 


\begin{tabular}{llcc}
\hline \hline & Description & Symbol & Value \\
\hline (i) & Oversupply of assets & $n_{a}-\left(n_{h}+n_{f}\right)$ & 0.08 \\
\hline \multirow{3}{*}{ (ii) } & $\begin{array}{l}\text { Impact of SBOs on fund valuation } \\
\text { (changing of } \lambda_{s} \text { from 0 to 699) }\end{array}$ & $\frac{\Delta v_{f n}}{v_{f n}}$ & $26.2 \%$ \\
& $\begin{array}{l}\text { Sensitivity of fund value to operations } \\
\text { Sensitivity of fund value to liquidity shocks }\end{array}$ & $\frac{\partial \log v_{f n}}{\partial \log u_{f}}$ & 4.22 \\
\hline (iii) & $\begin{array}{l}\text { Impact of PE entry on fund valuation } \\
\left.\text { (changing the number of funds from } n_{f} \text { to } 2 n_{f}\right)\end{array}$ & $\frac{\Delta v_{f n}}{v_{f n}}$ & 0.52 \\
\hline & $\begin{array}{l}\text { Impact of PE entry on transaction prices } \\
\left.\text { (changing the number of funds from } n_{f} \text { to } 2 n_{f}\right)\end{array}$ & $\frac{\Delta P_{l o-h n}}{P_{l o-h n}}$ & $-0.9 \%$ \\
\hline (iv) & $\begin{array}{l}\text { Welfare gain by M\&As and PEs } \\
\text { (relative to the welfare with no M\&A or PE) }\end{array}$ & $\frac{W-\underline{W}}{\underline{W}}$ & $13.6 \%$ \\
& $\begin{array}{l}\text { Welfare gain by M\&As and PEs } \\
\text { (relative to the maximum welfare gain) }\end{array}$ & $\frac{W-\underline{W}}{\bar{W}-\underline{W}}$ & $92.1 \%$ \\
Corporate sector's share of the welfare gain & $\frac{W{ }_{c}-\underline{W}}{W-\underline{W}}$ & $73.3 \%$ \\
\hline
\end{tabular}

Table 5: Calibration Results

the number of assets: $n_{a}>n_{h}+n_{f}{ }^{31}$ The result, together with large meeting rates, suggests that the M\&A middle market is close to Case $\mathrm{C}$ of the fast-search market (Proposition 8).

Second, SBOs significantly improve fund valuations, as explained with Figure 2. PE fund values with SBOs are $26.2 \%$ higher than without SBOs, highlighting the importance of complementarities among PE funds. While we acknowledge the criticism against SBOs, overall it is because of (and not in spite of) SBOs that PE funds generate high returns. An improvement in firms' operation $u_{f}$ by $1 \%$ leads to a significant $4.22 \%$ increase in fund value $v_{f n}$. However, a similar improvement of $u_{e}$ is attenuated by a vibrant SBO market and has a negligible influence on fund value: sensitivity is 0.52 . We find that, if PE funds cannot improve operations of acquired firms (e.g., $u_{l}=u_{f} \geq u_{e}$ ), then their intermediation would not be profitable, in spite of their search advantages $\left(\lambda_{f}>\lambda_{c}\right)$.

Third, when the number of PE funds increases, the percentage of SBOs relative to the total fund exits increases (Figure 3), which is a pattern observed in the market ${ }^{32}$ Doubling

\footnotetext{
${ }^{31}$ The calibration result on the oversupply of assets is not sensitive to our choice of $n_{a}$. For example, with $n_{a}$ equal to 0.25 or 0.75 , the oversupply $n_{a}-\left(n_{h}+n_{f}\right)$ is also approximately 0.08 .

${ }^{32}$ The share of firms sold by PE funds through SBOs has increased from 13\% in the 1980s, $19 \%$ in 2009,
} 
the number of PE funds would actually increase the average fund value by $0.7 \%$, suggesting a stronger role played by the complementarities among funds than the competitive effect. Doubling the number of funds leads to only a $0.9 \%$ decrease in transaction prices in the corporate M\&A market. This is because more purchases by funds empower corporate sellers and more assets sold by funds increases corporate buyers' bargaining position.

Finally, the welfare gain by the M\&A with PE market is $13.6 \%$, relative to the autarkic situation welfare $\underline{W}$ with no M\&A or PE intermediation (see page 18). This welfare gains by M\&A and PE intermediation attains $92.1 \%$ of the best possible welfare gains $(\bar{W}-\underline{W})$. The fraction is lower than the gain in OTC markets for municipal bonds as described in Hugonnier, Lester, and Weill (2018), likely due to higher search frictions in the M\&A market compared to the municipal bond markets. The corporations' percentage share of this welfare gain is $73.3 \%$, which leaves $26.7 \%$ of the gain to PE funds. This share for PE funds is large relative to the small number of PE funds.

\section{Conclusion}

We provide a search-based model of the M\&A market with PE intermediation. PE funds in our model intermediate between corporate buyers and sellers due to their ability to add operational value to portfolio firms. While SBOs are sometimes criticized as detrimental to fund investors, our model shows that PE funds generate high returns because of SBOs. This result offers a novel mechanism to explain persistent PE funds' returns in the M\&A market despite increased competition. Our paper suggests that a well-lubricated private market can partly explain the recent shift in firm ownership from public to private, predicated by Jensen (1991). Lower liquidity costs in a market for buying and selling private firms help make PE ownership a form of governance that may indeed eclipse public companies.

to $42 \%$ in 2017. IPOs, an alternative route to sell a portfolio firm, have declined from $277 /$ year in 1987-2007 to $98 /$ year in $2008-2017$. 


\section{A Proofs for Section 3}

\section{A.1 Proof of Lemma 1:}

Take any M\&A with PE market $\theta=(n, r, u, \rho, \lambda)$ with $n_{f}>0$. Let $\mu \in \mathbb{R}_{+}^{\mathcal{T}}$ be a steady-state solution of $P(\theta)$.

First, we show that $\mu_{h o}>0$ and $\mu_{l o}>0$. It is clear that $\mu_{h o}=0$ if and only if $\mu_{l o}=0$. If $\mu_{h o}=0$, then $\mu_{l o}=0$, as only $h o$-type corporations flow in type $l o$; conversely, if $\mu_{l o}=0$, then $\mu_{h o}=0$ as the inflow to the type $l o$ must be zero. Suppose, toward contradiction, that $\mu_{h o}=\mu_{l o}=0$. That is, all corporations are non-owners. The in-flow from $h n$-type corporations to type ho must be zero, so it must be that $\mu_{f_{o}}=\mu_{f e}=0$. Then, $\mu_{h o}+\mu_{l o}+\mu_{f o}+\mu_{f e}=0$, a contradiction to $n_{a}>0$.

Second, we show that $\mu_{l n}>0$ and $\mu_{h n}>0$. As before, it is clear that $\mu_{l n}=0$ if and only if $\mu_{h n}=0$. If $\mu_{l n}=0$, then $\mu_{h n}=0$ as only $l n$-type corporations can flow in type $h n$; conversely, if $\mu_{h n}=0$, then $\mu_{l n}=0$ as the inflow to the type $h n$ must be zero. Suppose, toward contradiction, that $\mu_{l n}=\mu_{h n}=0$. That is, all corporations are owners, which implies that some PE funds are non-owners: $\mu_{f n}=n_{c}+n_{f}-n_{a}>0$. Since $\lambda_{f} \mu_{l o} \mu_{f n}>0$, some lo-type corporations change their types and flow into type $\ln$ by trading with PE funds, a contradiction to $\mu_{l n}=0$.

Lastly, we consider PE funds. Suppose that $\mu_{f n}=0$. As the inflow to type $f_{o}$ becomes zero, it must be that $\mu_{f o}=0$, which in turn leads to no inflow by liquidity shocks to type fe: i.e., $\mu_{f e}=0$. Such case contradicts to $n_{f}>0$. When $\mu_{f n}>0$, given strictly positive population $\mu_{l o}$, the inflow of type- $f n$ funds to type $f_{o}$ is strictly positive: $\lambda_{f} \mu_{l o} \mu_{f n}>0$. As such, $\mu_{f o}>0$, which in turn creates a strictly positive inflow by liquidity shocks to type $f e$ : $\mu_{f e}>0$.

\section{A.2 Proof for Part 1 of Proposition 1}

We reduce the number of variables and population equations in $P(\theta)$ by imposing some necessary conditions for a steady-state solution. Note that any steady-state solution $\mu$ must satisfy $\mu_{h o}+\mu_{h n}=n_{h} \equiv \frac{\rho_{u}}{\rho_{u}+\rho_{d}}$ and $\mu_{l o}+\mu_{l n}=n_{l} \equiv \frac{\rho_{d}}{\rho_{u}+\rho_{d}}$ (which we can obtain by adding $\mu$-ho and $\mu$-hn , or $\mu$-lo and $\mu$-ln , and apply $\left.n_{c}=1\right)$. If we substitute $\mu_{h o}=n_{h}-\mu_{h n}$ 
and $\mu_{l n}=n_{l}-\mu_{l o}$ into $\mu$-ho $-\mu$-fe , then we are left with the following three linearly independent equations ${ }^{33}$

$$
\begin{array}{rlr}
\left(\lambda_{c} \mu_{h n}+\lambda_{f} \mu_{f n}\right) \mu_{l o}+\rho_{u} \mu_{l o}-\rho_{d} \mu_{h o}=0, & (\text { from } \mu-\mathrm{lo}) \\
\left(\lambda_{c} \mu_{l o}+\lambda_{f} \mu_{f o}+\lambda_{f} \mu_{f e}\right) \mu_{h n}+\rho_{d} \mu_{h n}-\rho_{u} \mu_{l n}=0, & (\text { from } \mu-\mathrm{hn}) \\
-\left(\lambda_{f} \mu_{h n}+\lambda_{s} \mu_{f n}\right) \mu_{f e}+\rho_{e} \mu_{f o}=0 . & (\text { from } \mu-\mathrm{fe}))
\end{array}
$$

We re-write the first two equations with respect to $\mu_{l o}$ and $\mu_{h n}$. Since

$$
\begin{aligned}
& \mu_{f o}+\mu_{f e}=n_{a}-\mu_{h o}-\mu_{l o}=n_{a}-\left(n_{h}-\mu_{h n}\right)-\mu_{l o} \text { and } \\
& \mu_{f n}=n_{f}-\left(\mu_{f o}+\mu_{f e}\right)=n_{f}-n_{a}+n_{h}-\mu_{h n}+\mu_{l o},
\end{aligned}
$$

we have

$$
\begin{aligned}
\left(\lambda_{c} \mu_{h n}+\lambda_{f}\left(n_{f}-n_{a}+n_{h}-\mu_{h n}+\mu_{l o}\right)\right) \mu_{l o}+\rho_{u} \mu_{l o}-\rho_{d}\left(n_{h}-\mu_{h n}\right) & =0 \\
\left(\lambda_{c} \mu_{l o}+\lambda_{f}\left(n_{a}-n_{h}+\mu_{h n}-\mu_{l o}\right)\right) \mu_{h n}+\rho_{d} \mu_{h n}-\rho_{u}\left(n_{l}-\mu_{l o}\right) & =0 .
\end{aligned}
$$

The idea is to focus on the supply $\left(\mu_{l o}\right)$ and demand $\left(\mu_{h n}\right)$ of assets in the corporate-M\&A market. An increase of supply $\mu_{l o}$ (or demand $\mu_{h n}$ ) of assets in the corporate M\&A market increases the outflow of agents from both supply and demand $\left(\lambda_{c} \mu_{l o} \mu_{h n}\right)$, which in turn increases the number of agents that are rightly holding or not-holding assets $\left(\mu_{h o}\right.$ and $\left.\mu_{l n}\right)$. This increased population of $\mu_{h o}$ and $\mu_{l n}$ increases the in-flow to supply $\left(\rho_{d} \mu_{h o}\right)$ and to demand $\left(\rho_{u} \mu_{l n}\right)$ and result in larger aggregate supply $\mu_{l o}$ and demand $\mu_{h n}$ of assets in the corporate-M\&A market. As such, when the population of PE funds are restricted to satisfy the constraints (5) and (6), a unique supply $\mu_{l o}$ (or demand $\mu_{h n}$ ) would satisfy the constraint $\mu_{l o}+\mu_{h n}+\mu_{l n}+\mu_{h o}=n_{c}=1$, by Intermediate Value Theorem.

We show below that there exists a unique solution $\left(\mu_{l o}, \mu_{h n}\right)$ of (7)-(8) such that (i)

\footnotetext{
${ }^{33}$ Any other equation in $P(\theta)$ is redundant, as it depends linearly on $(\mu$-lo,$\quad \mu$-hn $)$, and $\mu$-fe . Each sum of the right-hand sides of $\mu$-ho and $\mu$-hn , or $\mu$-lo and $\mu$-ln equals zero, which allow us to delete $\mu$-ho and $\mu-\ln$ without changing the solution set. The sum of the right-hand sides of $\mu$-fn,$\quad \mu$-for, and $\mu$-fe equals zero, so we can delete $\mu$-fn $)$. Last, the sum of the right-hand sides of $\mu$-ho, $\mu$-lo, $\mu$-fo, and $\mu$-fe equals zero, so we can delete $\mu$-fo.
} 
$0 \leq \mu_{l o} \leq n_{l}$, (ii) $0 \leq \mu_{h n} \leq n_{h}$, and (iii) $n_{a}-n_{f}-n_{h} \leq \mu_{l o}-\mu_{h n} \leq n_{a}-n_{h}$ (for $0 \leq \mu_{f n} \leq n_{f}$ ). Then, we can uniquely determine other population measures by $\mu_{h o}=n_{h}-\mu_{h n}, \mu_{l n}=n_{l}-\mu_{l o}$, and

$$
\mu_{f n}=n_{f}-n_{a}+n_{h}-\mu_{h n}+\mu_{l o} .
$$

We find the last two populations $\left(\mu_{f e}, \mu_{f o}\right)$ by solving

$$
\begin{aligned}
& \mu_{f o}=-\mu_{f e}+\left(n_{f}-\mu_{f n}\right), \quad\left(\text { from } \mu_{f n}+\mu_{f o}+\mu_{f e}=n_{f}\right) \\
& \mu_{f o}=\frac{\lambda_{f} \mu_{h n}+\lambda_{s} \mu_{f n}}{\rho_{e}} \mu_{f e} . \quad(\text { from } \mu-\text { fe })
\end{aligned}
$$

The unique solution is

$$
\mu_{f e}=\frac{\rho_{e}\left(n_{f}-\mu_{f n}\right)}{\lambda_{f} \mu_{h n}+\lambda_{s} \mu_{f n}+\rho_{e}}, \quad \text { and } \quad \mu_{f o}=\frac{\left(\lambda_{f} \mu_{h n}+\lambda_{s} \mu_{f n}\right)\left(n_{f}-\mu_{f n}\right)}{\lambda_{f} \mu_{h n}+\lambda_{s} \mu_{f n}+\rho_{e}}
$$

Therefore, it remains to prove the following claim:

Claim 1. Let

$$
X(\theta) \equiv\left\{\left(x_{1}, x_{2}\right) \in \mathbb{R}^{2}: 0 \leq x_{1} \leq n_{l}, 0 \leq x_{2} \leq n_{h}, 0 \leq g_{f n}(x) \leq n_{f}\right\} .
$$

where $g_{f n}(x) \equiv n_{a}-n_{h}+x_{2}-x_{1}$. Also, define $F \equiv\left(F_{l o}, F_{h n}\right): \mathbb{R}^{2} \rightarrow \mathbb{R}^{2}$ by

$$
\begin{aligned}
& F_{l o}(x) \equiv\left(\lambda_{c} x_{2}+\lambda_{f}\left(n_{f}-g_{f n}(x)\right)\right) x_{1}+\rho_{u} x_{1}-\rho_{d}\left(n_{h}-x_{2}\right), \\
& F_{h n}(x) \equiv\left(\lambda_{c} x_{1}+\lambda_{f} g_{f n}(x)\right) x_{2}+\rho_{d} x_{2}-\rho_{u}\left(n_{l}-x_{1}\right) .
\end{aligned}
$$

Then, there exists a unique solution of $F(x)=0$ in $X(\theta)$.

We apply the Poincare-Hopf index theorem, a version in Simsek, Ozdaglar, and Acemoglu (2007, p.194); see also Hirsch (2012). 
First, $X(\theta)$ is non-empty, compact, and convex ${ }^{34}$ The boundary of $X(\theta)$ is

$$
\partial X(\theta) \equiv\left\{\left(x_{1}, x_{2}\right) \in X(\theta): x_{1}=0, x_{1}=n_{l}, x_{2}=0, x_{2}=n_{h}, g_{f n}(x)=0 \text {, or } g_{f n}(x)=n_{f}\right\}
$$

Second, the function $F(x)$ is continuously differentiable at every $x \in \mathbb{R}^{2}$.

Third, we show that the determinant of the Jacobian matrix of $F$ is strictly positive for every interior point of $X(\theta)$. For each $x \in \mathbb{R}^{2}$,

$$
\begin{aligned}
\nabla F(x) & \equiv\left[\begin{array}{ll}
\frac{\partial F_{l o}}{\partial x_{1}} & \frac{\partial F_{l o}}{\partial x_{2}} \\
\frac{\partial F_{h n}}{\partial x_{1}} & \frac{\partial F_{h n}}{\partial x_{2}}
\end{array}\right] \\
& =\left[\begin{array}{cc}
\left(\lambda_{c} x_{2}+\lambda_{f}\left(n_{f}-g_{f n}(x)\right)\right)+\lambda_{f} x_{1}+\rho_{u} & \left(\lambda_{c}-\lambda_{f}\right) x_{1}+\rho_{d} \\
\left(\lambda_{c}-\lambda_{f}\right) x_{2}+\rho_{u} & \left(\lambda_{c} x_{1}+\lambda_{f} g_{f n}(x)\right)+\lambda_{f} x_{2}+\rho_{d}
\end{array}\right]
\end{aligned}
$$

For any interior point $x \in X(\theta) \backslash \partial X(\theta)$,

$$
\begin{aligned}
\operatorname{det}(\nabla F(x)) \geq & \left(\lambda_{c} x_{2}+\lambda_{f} x_{1}\right)\left(\lambda_{c} x_{1}+\lambda_{f} x_{2}\right)+\rho_{u} \lambda_{c} x_{1}+\rho_{d} \lambda_{c} x_{2} \\
& -\left(\lambda_{c}-\lambda_{f}\right)^{2} x_{1} x_{2}-\left(\lambda_{c}-\lambda_{f}\right)\left(\rho_{d} x_{2}+\rho_{u} x_{1}\right) \\
= & \lambda_{c} \lambda_{f}\left(x_{1}^{2}+x_{2}^{2}\right)+2 \lambda_{c} \lambda_{f} x_{1} x_{2}+\lambda_{f}\left(\rho_{d} x_{2}+\rho_{u} x_{1}\right) \\
> & 0 .
\end{aligned}
$$

Last, we show that, for every boundary point $x \in \partial X(\theta)$, the vector $F(x) \in \mathbb{R}^{2}$ points strictly outward of $X(\theta)$. We partition the boundary $\partial X(\theta)$ into six faces (i.e., flat surfaces) of $X(\theta)$. For each face, we find an outward normal vector $\mathbf{n} \in \mathbb{R}^{2}$ and show that the angle between $\mathbf{n}$ and $F(x)$ is acute (i.e., $\leq 90)$ at any point $x$ in the face:

1. $\left(x_{1}=0\right.$ and $\left.0 \leq x_{2}<n_{h}\right) \mathbf{n}=(-1,0)$ is an outward normal vector, and $\mathbf{n} \cdot F(x)=$ $\rho_{d}\left(n_{h}-x_{2}\right)>0$.

2. $\left(x_{2}=0\right.$ and $\left.0 \leq x_{1}<n_{l}\right) \mathbf{n}=(0,-1)$ is an outward normal vector, and $\mathbf{n} \cdot F(x)=$ $\rho_{u}\left(n_{l}-x_{1}\right)>0$.

${ }^{34}$ The Poincare-Hopf index theorem also requires $X(\theta)$ to be a 2-dimensional smooth manifold, which a reader can easily verify by applying the identify function to the definition of a smooth manifold in Simsek, Ozdaglar, and Acemoglu (2007, p.193). 
3. $\left(x_{1}=n_{l}\right.$ and $\left.0 \leq x_{2} \leq n_{h}\right) \mathbf{n}=(1,0)$ is an outward normal vector, and

$$
\begin{aligned}
\mathbf{n} \cdot F(x) & =\left(\lambda_{c} x_{2}+\lambda_{f}\left(n_{f}-g_{f n}(x)\right)\right) n_{l}+\rho_{u} n_{l}-\rho_{d} n_{h}+\rho_{d} x_{2} \\
& \geq\left(\lambda_{c} x_{2}+\lambda_{f}\left(n_{f}-g_{f n}(x)\right)\right) n_{l} \quad\left(\text { as } \rho_{u} n_{l}=\rho_{d} n_{h}\right) \\
& \geq \min \left\{\lambda_{c} x_{2} n_{l}, \lambda_{f}\left(n_{f}-g_{f n}(x)\right) n_{l}\right\} .
\end{aligned}
$$

As either $x_{2}>0$ or $x_{2}=0$, we have $n_{f}-g_{f n}(x)=n_{c}+n_{f}-n_{a}>0$, and $\mathbf{n} \cdot F(x)>0$.

4. $\left(x_{2}=n_{h}\right.$ and $\left.0 \leq x_{1} \leq n_{l}\right) \mathbf{n}=(0,1)$ is an outward normal vector, and

$$
\begin{aligned}
\mathbf{n} \cdot F(x) & =\left(\lambda_{c} x_{1}+\lambda_{f} g_{f n}(x)\right) n_{h}+\rho_{d} n_{h}-\rho_{u} n_{l}+\rho_{u} x_{1} \\
& \geq \min \left\{\lambda_{c} x_{1} n_{h}, \lambda_{f} g_{f n}(x) n_{h}\right\}
\end{aligned}
$$

As either $x_{1}>0$ or $x_{1}=0$, we have $g_{f n}(x)=n_{a}>0$, and $\mathbf{n} \cdot F(x)>0$.

5. $\left(g_{f n}(x)=0\right.$ and $\left.x_{1}>0\right) \mathbf{n}=(1,-1)$ is an outward normal vector, and $\mathbf{n} \cdot F(x)=$ $F_{l o}(x)-F_{h n}(x)=\lambda_{f} n_{f} x_{1}>0$.

6. $\left(g_{f n}(x)=n_{f}\right.$ and $\left.x_{2}>0\right) \mathbf{n}=(-1,1)$ is an outward normal vector, and $\mathbf{n} \cdot F(x)=$ $F_{h n}(x)-F_{l o}(x)=\lambda_{f} n_{f} x_{2}>0$.

We are ready to apply the Poincare-Hopf index theorem in Simsek, Ozdaglar, and Acemoglu (2007, p.194). The Euler characteristic of $X(\theta)$ is 1; see their definition on p.193 for the case of non-empty and convex sets. Claim 1 follows immediately from the index theorem, which completes the proof for Part 1 of Proposition 1.

\section{A.3 Proof for Part 2 of Proposition 1}

We want to show asymptotic stability of an equilibrium of continuous dynamic system. We first reduce the system $P(\theta)$. For any market $\theta$ and an initial condition $\mu(0)$, any dynamic 
solution $\mu:[0, \infty) \rightarrow \mathbb{R}^{\mathcal{T}}$ of the system $P(\theta)$ satisfies, for every $t \in[0, \infty)$,

$$
\begin{aligned}
\mu_{h o}(t)+\mu_{h n}(t)+\mu_{l o}(t)+\mu_{l n}(t) & =n_{c}(=1), \\
\mu_{f n}(t)+\mu_{f o}(t)+\mu_{f e}(t) & =n_{f}, \quad \text { and } \\
\mu_{h o}(t)+\mu_{l o}(t)+\mu_{f o}(t)+\mu_{f e}(t) & =n_{a} .
\end{aligned}
$$

As such, without changing the set of dynamic solutions, we can reduce the system $P(\theta)$ for $x(t) \equiv\left(\mu_{h o}(t), \mu_{h n}(t), \mu_{l o}(t), \mu_{f o}(t)\right)$ by

$$
\dot{x}=F(x) \equiv\left(F_{h o}(x), F_{h n}(x), F_{l o}(x), F_{f o}(x)\right),
$$

where

$$
\begin{aligned}
F_{h o}(x) & \equiv\left(\lambda_{c} \mu_{l o}+\lambda_{f} \mu_{f o}+\lambda_{f} \mu_{f e}(x)\right) \mu_{h n}-\rho_{d} \mu_{h o}+\rho_{u} \mu_{l o}, \\
F_{h n}(x) & \equiv-\left(\lambda_{c} \mu_{l o}+\lambda_{f} \mu_{f o}+\lambda_{f} \mu_{f e}(x)\right) \mu_{h n}-\rho_{d} \mu_{h n}+\rho_{u} \mu_{l n}(x), \\
F_{l o}(x) & \equiv-\left(\lambda_{c} \mu_{h n}+\lambda_{f} \mu_{f n}(x)\right) \mu_{l o}-\rho_{u} \mu_{l o}+\rho_{d} \mu_{h o}, \\
F_{f o}(x) & \equiv\left(\lambda_{f} \mu_{l o}+\lambda_{s} \mu_{f e}(x)\right) \mu_{f n}(x)-\lambda_{f} \mu_{h n} \mu_{f o}-\rho_{e} \mu_{f o},
\end{aligned}
$$

and

$$
\begin{aligned}
\mu_{l n}(x) & \equiv 1-\mu_{h o}-\mu_{h n}-\mu_{l o} \\
\mu_{f e}(x) & \equiv n_{a}-\mu_{h o}-\mu_{l o}-\mu_{f o} \\
\mu_{f n}(x) & \equiv n_{f}-\mu_{f o}-\mu_{f e}(x)=n_{f}-n_{a}+\mu_{h o}+\mu_{l o} .
\end{aligned}
$$

The above reduction of the system $P(\theta)$ does not change the set of dynamic solutions 35 If $\mu$ is a dynamic (either steady-state or not) solution of $P(\theta)$, then $x \equiv\left(\mu_{h o}, \mu_{h n}, \mu_{l o}, \mu_{f o}\right)$ solves $F(x ; \theta)=0$; conversely, for any dynamic solution $x$ of $F(x ; \theta)=0$, we can find a dynamic solution $\mu$ of $P(\theta)$, from $x$ and the induced $\mu_{l n}, \mu_{f e}$, and $\mu_{f n}$. Therefore, a

\footnotetext{
${ }^{35}$ In the proof of Part 1 of Proposition 1 , we reduced $F(x ; \theta)=0$ further as a system of only two equations in Claim 1. The reduction requires $\mu_{h n}+\mu_{h o}=n_{h} \equiv \frac{\rho_{u}}{\rho_{u}+\rho_{d}}$ and $\mu_{l o}+\mu_{l n}=n_{l} \equiv \frac{\rho_{d}}{\rho_{u}+\rho_{d}}$, which hold in a steady-state but may not hold on a path of $\mu(t)$ after a perturbation.
} 
dynamic solution $\mu$ of $P(\theta)$ is asymptotically stable if and only if $x \equiv\left(\mu_{h o}, \mu_{h n}, \mu_{l o}, \mu_{f o}\right)$ is asymptotically stable.

A steady-state solution $x$ of $F(x ; \theta)=0$ is asymptotically stable if all eigenvalues of the Jacobian matrix of $F(x ; \theta)$ at the steady-state solution $x$ have strictly negative real parts (Hirsch, 2012). The Jacobian matrix is

$$
\begin{aligned}
& \nabla F(x) \equiv\left[\frac{\partial F_{i}(x)}{\partial x_{j}}\right]_{i, j \in\{h o, h n, l o, f o\}} \\
& =\left[\begin{array}{ccc|c}
-\lambda_{f} \mu_{h n}-\rho_{d} & \lambda_{c} \mu_{l o}+\lambda_{f} \mu_{f o}+\lambda_{f} \mu_{f e} & \left(\lambda_{c}-\lambda_{f}\right) \mu_{h n}+\rho_{u} & 0 \\
\lambda_{f} \mu_{h n}-\rho_{u} & -\left(\lambda_{c} \mu_{l o}+\lambda_{f} \mu_{f o}+\lambda_{f} \mu_{f e}\right)-\rho_{d}-\rho_{u} & \left(\lambda_{f}-\lambda_{c}\right) \mu_{h n}-\rho_{u} & 0 \\
-\lambda_{f} \mu_{l o}+\rho_{d} & -\lambda_{c} \mu_{l o} & -\lambda_{f}\left(\mu_{f n}+\mu_{l o}\right)-\lambda_{c} \mu_{h n}-\rho_{u} & 0 \\
\hline \lambda_{f} \mu_{l o}+\lambda_{s}\left(\mu_{f e}-\mu_{f n}\right) & -\lambda_{f} \mu_{f o} & \lambda_{f}\left(\mu_{f n}+\mu_{l o}\right)+\lambda_{s}\left(\mu_{f e}-\mu_{f n}\right) & -\lambda_{f} \mu_{h n}-\lambda_{s} \mu_{f n}-\rho_{e}
\end{array}\right]
\end{aligned}
$$

where we omit the dependency of $\mu_{f n}$ and $\mu_{f e}$ on $x$ to simplify the expression.

Due to the block structure, one eigenvalue is $-\lambda_{f} \mu_{h n}-\lambda_{s} \mu_{f n}-\rho_{e}<0$. The other eigenvalues are the eigenvalue of the $3 \times 3$ sub-matrix with the first three rows and columns. A direct calculation shows that the other three eigenvalues are also strictly negative, which completes the proof.

\section{A.4 Proof of Proposition 2}

First, we simplify expositions:

$$
\begin{aligned}
& g_{1} \equiv g_{f o-h n}=(1 / 2)\left(v_{h o}+v_{f n}-v_{f o}-v_{h n}\right), \\
& g_{2} \equiv g_{l o-f n}=(1 / 2)\left(v_{f o}+v_{l n}-v_{l o}-v_{f n}\right), \\
& g_{3} \equiv g_{f e-f n}=(1 / 2)\left(v_{f o}+v_{f n}-v_{f e}-v_{f n}\right)=(1 / 2)\left(v_{f o}-v_{f e}\right),
\end{aligned}
$$

so that

$$
\begin{aligned}
& g_{l o-h n}=(1 / 2)\left(v_{h o}+v_{l n}-v_{l o}-v_{h n}\right)=g_{2}+g_{1} \quad \text { and } \\
& g_{f e-h n}=(1 / 2)\left(v_{h o}+v_{f n}-v_{f e}-v_{h n}\right)=g_{1}+g_{3} .
\end{aligned}
$$


The matrix representations of the value equations $\mathrm{v-hn}-\mathrm{v}-\mathrm{fe}$ are:

$$
\begin{aligned}
& {\left[\begin{array}{l}
v_{h o} \\
v_{l o}
\end{array}\right]=\left[\begin{array}{cc}
r+\rho_{d} & -\rho_{d} \\
-\rho_{u} & r+\rho_{u}
\end{array}\right]^{-1}\left[\begin{array}{c}
u_{h} \\
u_{l}+\lambda_{c} \mu_{h n}\left(g_{1}+g_{2}\right)+\lambda_{f} \mu_{f n} g_{2}
\end{array}\right],} \\
& {\left[\begin{array}{l}
v_{h n} \\
v_{l n}
\end{array}\right]=\left[\begin{array}{cc}
r+\rho_{d} & -\rho_{d} \\
-\rho_{u} & r+\rho_{u}
\end{array}\right]^{-1}\left[\begin{array}{c}
\lambda_{c} \mu_{l o}\left(g_{1}+g_{2}\right)+\lambda_{f} \mu_{f o} g_{1}+\lambda_{f} \mu_{f e}\left(g_{1}+g_{3}\right) \\
0
\end{array}\right], \quad \text { and }} \\
& {\left[\begin{array}{l}
v_{f n} \\
v_{f o} \\
v_{f e}
\end{array}\right]=\frac{1}{r}\left[\begin{array}{c}
\lambda_{f} \mu_{l o} g_{2}+\lambda_{s} \mu_{f e} g_{3} \\
u_{f}+\lambda_{f} \mu_{h n} g_{1}-2 \rho_{e} g_{3} \\
u_{e}+\lambda_{f} \mu_{h n}\left(g_{1}+g_{3}\right)+\lambda_{s} \mu_{f n} g_{3}
\end{array}\right],}
\end{aligned}
$$

where the inverse matrix is well-defined: i.e., $\left(r+\rho_{d}\right)\left(r+\rho_{u}\right)-\rho_{d} \rho_{u}>0$. As in the case of $n_{f}=0$, we compute the gains $g_{1}, g_{2}$, and $g_{3}$. Then, the solution $v$ will be uniquely determined by the above matrix equations.

First,

$$
2 r g_{3}=r\left(v_{f o}-v_{f e}\right)=\left(u_{f}-u_{e}\right)-2 \rho_{e} g_{3}-\lambda_{f} \mu_{h n} g_{3}-\lambda_{s} \mu_{f n} g_{3},
$$

which results in

$$
g_{3}=\frac{u_{f}-u_{e}}{2 r+2 \rho_{e}+\lambda_{f} \mu_{h n}+\lambda_{s} \mu_{f n}}>0
$$

Next,

$$
\begin{aligned}
2\left(g_{1}+g_{2}\right) & =v_{h o}+v_{l n}-v_{l o}-v_{h n}=(1,-1) \cdot\left(v_{h o}-v_{h n}, v_{l o}-v_{l n}\right) \\
& =\left[\begin{array}{ll}
1 & -1
\end{array}\right]\left[\begin{array}{cc}
r+\rho_{d} & -\rho_{d} \\
-\rho_{u} & r+\rho_{u}
\end{array}\right]^{-1}\left[\begin{array}{c}
u_{h}-\lambda_{c} \mu_{l o}\left(g_{1}+g_{2}\right)-\lambda_{f} \mu_{f o} g_{1}-\lambda_{f} \mu_{f e}\left(g_{1}+g_{3}\right) \\
u_{l}+\lambda_{c} \mu_{h n}\left(g_{1}+g_{2}\right)+\lambda_{f} \mu_{f n} g_{2}
\end{array}\right] .
\end{aligned}
$$

Since

$$
\left[\begin{array}{ll}
1 & -1
\end{array}\right]\left[\begin{array}{cc}
r+\rho_{d} & -\rho_{d} \\
-\rho_{u} & r+\rho_{u}
\end{array}\right]^{-1}=\frac{1}{r+\rho_{u}+\rho_{d}}\left[\begin{array}{ll}
1 & -1
\end{array}\right]
$$


we have

$$
\begin{array}{r}
\left(2\left(r+\rho_{u}+\rho_{d}\right)+\lambda_{c}\left(\mu_{l o}+\mu_{h n}\right)\right)\left(g_{1}+g_{2}\right)+\lambda_{f}\left(\mu_{f o}+\mu_{f e}\right) g_{1}+\lambda_{f} \mu_{f n} g_{2} \\
=\left(u_{h}-u_{l}\right)-\lambda_{f} \mu_{f e} g_{3}
\end{array}
$$

On the other hand, by $\mathrm{v}-\mathrm{lo}), \mathrm{v}-\mathrm{ln}), \mathrm{v}-\mathrm{fn})$, and $(\mathrm{v}-\mathrm{fo})$,

$$
\begin{aligned}
2 r g_{2}= & r\left(v_{f o}-v_{f n}\right)-r\left(v_{l o}-v_{l n}\right) \\
= & \left(u_{f}+\lambda_{f} \mu_{h n} g_{1}-2 \rho_{e} g_{3}-\lambda_{f} \mu_{l o} g_{2}-\lambda_{s} \mu_{f e} g_{3}\right) \\
& -\left(u_{l}+\lambda_{c} \mu_{h n}\left(g_{1}+g_{2}\right)+\lambda_{f} \mu_{f n} g_{2}\right)+\rho_{u}\left(v_{h o}-v_{l o}+v_{l n}-v_{h n}\right) .
\end{aligned}
$$

As $v_{h o}-v_{l o}+v_{l n}-v_{h n}=2\left(g_{1}+g_{2}\right)$,

$$
\begin{array}{r}
\left(2 \rho_{u}+\lambda_{c} \mu_{h n}\right)\left(g_{1}+g_{2}\right)-\lambda_{f} \mu_{h n} g_{1}+\left(2 r+\lambda_{f} \mu_{l o}+\lambda_{f} \mu_{f n}\right) g_{2} \\
=\left(u_{f}-u_{l}\right)-\left(2 \rho_{e}+\lambda_{s} \mu_{f e}\right) g_{3} .
\end{array}
$$

The linear system of equations $(16)$ and $(17)$ is summarized as follows:

$$
\left[\begin{array}{cc}
c_{1}+\lambda_{f}\left(\mu_{f o}+\mu_{f e}\right) & c_{1}+\lambda_{f} \mu_{f n} \\
c_{2}-\lambda_{f} \mu_{h n} & c_{2}+2 r+\lambda_{f}\left(\mu_{l o}+\mu_{f n}\right)
\end{array}\right]\left[\begin{array}{l}
g_{1} \\
g_{2}
\end{array}\right]=\left[\begin{array}{c}
u_{h}-u_{l}-\lambda_{f} \mu_{f e} g_{3} \\
u_{f}-u_{l}-2 \rho_{e} g_{3}-\lambda_{s} \mu_{f e} g_{3}
\end{array}\right]
$$

where $c_{1} \equiv 2\left(r+\rho_{u}+\rho_{d}\right)+\lambda_{c}\left(\mu_{l o}+\mu_{h n}\right)>0$ and $c_{2} \equiv 2 \rho_{u}+\lambda_{c} \mu_{h n}>0$.

The determinant of the coefficient matrix is bounded below by

$$
2 r c_{1}+\lambda_{f} \mu_{f n}\left(c_{1}-c_{2}\right)>4 r^{2}+\lambda_{f} \mu_{f n}\left(2 r+2 \rho_{d}+\lambda_{c} \mu_{l o}\right)>0 .
$$

Thus, the above linear system has a unique solution $\left(g_{1}, g_{2}\right)$. This solution, together with $g_{3}$, determined the unique solution $v$ of $V(\theta)$. 


\section{A.5 Proof of Proposition 3}

A unique steady-state equilibrium exists if and only if the above solution $\left(g_{1}, g_{2}\right)$ is nonnegative:

$$
\begin{aligned}
g_{1} \equiv g_{f o-h n} \geq 0 \Longleftrightarrow & \left(c_{2}+2 r+\lambda_{f}\left(\mu_{l o}+\mu_{f n}\right)\right)\left(\left(u_{h}-u_{l}\right)-\lambda_{f} \mu_{f e} g_{3}\right) \\
& -\left(c_{1}+\lambda_{f} \mu_{f n}\right)\left(\left(u_{f}-u_{l}\right)-\left(2 \rho_{e}+\lambda_{s} \mu_{f e}\right) g_{3}\right) \geq 0 . \\
g_{2} \equiv g_{l o-f n} \geq 0 \Longleftrightarrow & -\left(c_{2}-\lambda_{f} \mu_{h n}\right)\left(\left(u_{h}-u_{l}\right)-\lambda_{f} \mu_{f e} g_{3}\right) \\
& +\left(c_{1}+\lambda_{f}\left(\mu_{f o}+\mu_{f e}\right)\right)\left(\left(u_{f}-u_{l}\right)-\left(2 \rho_{e}+\lambda_{s} \mu_{f e}\right) g_{3}\right) \geq 0
\end{aligned}
$$

Note that both expressions depends on the steady-state population measure $\mu$.

\section{A.6 Proof of Lemma 2}

For Part 1:

$$
\begin{aligned}
& 2\left(p_{f o-h n}-p_{f e-h n}\right)=v_{f o}-v_{f e}=2 g_{f e-f n} \geq 0, \\
& 2\left(p_{f e-h n}-p_{f e-f n}\right)=\left(v_{h o}-v_{h n}\right)-\left(v_{f o}-v_{f n}\right)=2 g_{f o-h n} \geq 0 .
\end{aligned}
$$

For Part 2:

$$
\begin{aligned}
2\left(p_{f o-h n}-p_{l o-h n}\right) & =\left(v_{h o}-v_{h n}+v_{f o}-v_{f n}\right)-\left(v_{l o}-v_{l n}+v_{f o}-v_{f n}\right) \\
& =\left(v_{h o}-v_{h n}\right)-\left(v_{l o}-v_{l n}\right)=2 g_{l o-h n} \geq 0, \\
2\left(p_{l o-h n}-p_{l o-f n}\right) & =\left(v_{h o}-v_{h n}\right)-\left(v_{f o}-v_{f n}\right)=2 g_{f o-h n} \geq 0 .
\end{aligned}
$$

\section{A.7 Proof of Lemma 3}

Take any market $\theta$ with a unique steady-state equilibrium $(\mu, v)$. Define

$$
V_{c}(\theta) \equiv \sum_{i \in \mathcal{T}_{c}} \mu_{i} v_{i}, \quad V_{f}(\theta) \equiv \sum_{i \in \mathcal{T}_{f}} \mu_{i} v_{i}, \quad \text { and } \quad V(\theta) \equiv \sum_{i \in \mathcal{T}} \mu_{i} v_{i}
$$


First, we prove $r V_{c}(\theta)=W_{c}(\theta)$. From $(\mathrm{v}-\mathrm{hn})-(\mathrm{v}-\mathrm{ln})$,

$$
\begin{aligned}
r V_{c}(\theta) \equiv & r\left(\mu_{h o} v_{h o}+\mu_{h n} v_{h n}+\mu_{l o} v_{l o}+\mu_{l n} v_{l n}\right) \\
= & \mu_{h o}\left(u_{h}+\rho_{d}\left(v_{l o}-v_{h o}\right)\right)+\mu_{h n}\left(\lambda_{c} \mu_{l o} g_{l o-h n}+\lambda_{f} \mu_{f o} g_{f o-h n}+\lambda_{f} \mu_{f e} g_{f e-h n}+\rho_{d}\left(v_{l n}-v_{h n}\right)\right) \\
& +\mu_{l o}\left(u_{l}+\lambda_{c} \mu_{h n} g_{l o-h n}+\lambda_{f} \mu_{f n} g_{l o-f n}+\rho_{u}\left(v_{h o}-v_{l o}\right)\right)+\mu_{l n} \rho_{u}\left(v_{h n}-v_{l n}\right) .
\end{aligned}
$$

We rewrite the above expression in terms of the corporations' expected values and their payments to or received from PE funds. That is, we substitute $g_{f o-h n}=v_{h o}-v_{h n}-p_{f o-h n}$, $g_{f e-h n}=v_{h o}-v_{h n}-p_{f e-h n}, g_{l o-f n}=p_{l o-f n}-v_{l o}-v_{l n}$, and $g_{l o-h n}=(1 / 2)\left(v_{h o}+v_{l n}-v_{l o}+v_{h n}\right)$ and obtain

$$
\begin{aligned}
r V_{c}(\theta)-W_{c}(\theta)= & \left(\rho_{u} \mu_{l o}-\rho_{d} \mu_{h o}\right)\left(v_{h o}-v_{l o}\right)+\left(\rho_{u} \mu_{l n}-\rho_{u} \mu_{h n}\right)\left(v_{h n}-v_{l n}\right) \\
& +\mu_{h n}\left(\lambda_{c} \mu_{l o}+\lambda_{f} \mu_{f o}+\lambda_{f} \mu_{f e}\right)\left(v_{h o}-v_{h n}\right)+\mu_{l o}\left(\lambda_{c} \mu_{h n}+\lambda_{f} \mu_{f n}\right)\left(v_{l n}-v_{l o}\right) .
\end{aligned}
$$

The combined coefficient of $v_{h o}$ on the right-hand side of the above equation is $-\rho_{d} \mu_{h o}+$ $\rho_{u} \mu_{l o}+\mu_{h n}\left(\lambda_{c} \mu_{l o}+\lambda_{f} \mu_{f o}+\lambda_{f} \mu_{f e}\right)$, which equals the right-hand side of the population equation $\mu$-ho, so it is zero. We can similarly verify that the combined coefficient of $v_{i}$ for $i=h n, l o, l n$ are all equal to zero. Therefore, $r V_{c}(\theta)=W_{c}(\theta)$.

Second, we obtain from all population equations $\mu$-hn $-\mu$-fe that

$$
\begin{aligned}
r V(\theta)-W(\theta)= & \left(\rho_{u} \mu_{l o}-\rho_{d} \mu_{h o}\right)\left(v_{h o}-v_{l o}\right)+\left(\rho_{u} \mu_{l n}-\rho_{d} \mu_{h n}\right)\left(v_{h n}-v_{l n}\right)+\rho_{e} \mu_{f o}\left(v_{f e}-v_{f o}\right) \\
& +\mu_{h n}\left(\lambda_{c} \mu_{l o}+\lambda_{f} \mu_{f o}+\lambda_{f} \mu_{f e}\right)\left(v_{h o}-v_{h n}\right)+\mu_{l o}\left(\lambda_{c} \mu_{h n}+\lambda_{f} \mu_{f n}\right)\left(v_{l n}-v_{l o}\right) \\
& +\left(\left(\lambda_{f} \mu_{l o}+\lambda_{s} \mu_{f e}\right) \mu_{f n}-\lambda_{f} \mu_{h n} \mu_{f o}\right) v_{f o} \\
& +\lambda_{f}\left(\mu_{h n} \mu_{f o}+\mu_{h n} \mu_{f e}-\mu_{l o} \mu_{f n}\right) v_{f n}-\left(\lambda_{f} \mu_{h n}+\lambda_{s} \mu_{f n}\right) \mu_{f e} v_{f e} .
\end{aligned}
$$

As before, we can verify that the combined coefficients of $v_{i}$ for each $i \in \mathcal{T}$ equals the right-hand side of the type's population equation, so it is zero. As such, $r V(\theta)=W(\theta)$.

Lastly, $r V_{f}(\theta)=r\left(V(\theta)-V_{c}(\theta)\right)=W(\theta)-W_{c}(\theta)=W_{f}(\theta)$. 


\section{B Proofs for Section 4}

\section{B.1 Proof of Proposition 4}

Let $(\mu(\theta), v(\theta))$ be the unique steady-state solution of population and value for each market $\theta$. We compute the comparative static derivatives with respect to $\lambda_{s}$. It is intuitive that the unique steady-state measure of each corporate type $\left(\mu_{i}\right)_{i \in \mathcal{T}_{c}}$ and the measure $\mu_{f n}$ are independent of $\lambda_{s}$. Through a SBO, one PE fund changes its type from $f e$ to $f n$, replacing another fund of type changed from $f n$ to $f_{o}$.

To confirm the intuition, from the proof of Proposition 1, take the unique steady-state solution $x(\theta) \equiv\left(\mu_{l o}(\theta), \mu_{h n}(\theta)\right)$ of $F(x) \equiv\left(F_{l o}(x), F_{h n}(x)\right)=0$, where

$$
\begin{aligned}
& F_{l o}(x) \equiv\left(\lambda_{c} x_{2}+\lambda_{f}\left(n_{f}-g_{f n}(x)\right)\right) x_{1}+\rho_{u} x_{1}-\rho_{d}\left(n_{h}-x_{2}\right), \\
& F_{h n}(x) \equiv\left(\lambda_{c} x_{1}+\lambda_{f} g_{f n}(x)\right) x_{2}+\rho_{d} x_{2}-\rho_{u}\left(n_{l}-x_{1}\right) .
\end{aligned}
$$

By Implicit function theorem, $x(\theta)$ is differentiable in $\lambda_{s}$, and

$$
\frac{\partial x(\theta)}{\partial \lambda_{s}}=-\left[\nabla_{x} F(x(\theta) ; \theta)\right]^{-1} \frac{\partial F(x(\theta) ; \theta)}{\partial \lambda_{s}}
$$

We denoted the domain of $F(x)$ by $X(\theta)$ in the proof of Claim 1 . The unique solution $x(\theta)$ of $F(x ; \theta)=0$ is an interior point of $X(\theta)$, as shown in Lemma 1 for the case of $n_{f}>0$ and in the proof for Part 1 of Proposition 1 for the case of $n_{f}=0$. Thus, we have shown in the proof of Claim 1, the Jacobian matrix $\nabla F(x)$ at the unique solution $x(\theta)$ is invertible.

It follows from

$$
\frac{\partial F(x(\theta) ; \theta)}{\partial \lambda_{s}}=\left[\begin{array}{l}
0 \\
0
\end{array}\right]
$$

that

$$
\frac{\partial x(\theta)}{\partial \lambda_{s}}=\left[\begin{array}{l}
\partial \mu_{l o}(\theta) / \partial \lambda_{s} \\
\partial \mu_{h n}(\theta) / \partial \lambda_{s}
\end{array}\right]=\left[\begin{array}{l}
0 \\
0
\end{array}\right] .
$$

For other types, it follows from $\mu_{h o}(\theta)+\mu_{h n}(\theta)=n_{h}$ and $\mu_{l o}(\theta)+\mu_{l n}(\theta)=n_{l}$ that $\frac{\partial \mu_{l n}(\theta)}{\partial \lambda_{s}}=$ $\frac{\partial \mu_{h o}(\theta)}{\partial \lambda_{s}}=0$, and from $\mu_{h o}(\theta)+\mu_{l o}+\left(n_{f}-\mu_{f n}(\theta)\right)=n_{f}$ that $\frac{\partial \mu_{f n}(\theta)}{\partial \lambda_{s}}=0$. Lastly, from (10) 
and $\mu_{f o}+\mu_{f e}+\mu_{f n}=n_{f}$,

$$
\frac{\partial \mu_{f e}(\theta)}{\partial \lambda_{s}}=\frac{-\rho_{e}\left(n_{f}-\mu_{f n}(\theta)\right) \mu_{f n}(\theta)}{\left(\rho_{e}+\lambda_{f} \mu_{h n}(\theta)+\lambda_{s} \mu_{f n}(\theta)\right)^{2}}=-\frac{\partial \mu_{f o}(\theta)}{\partial \lambda_{s}}
$$

From the definition of $g_{3}$ on p. 41 and Equation 15 .

$$
v_{f o}-v_{f e}=2 g_{f e-f n}=2 g_{3}=\frac{2\left(u_{f}-u_{e}\right)}{2 r+2 \rho_{e}+\lambda_{f} \mu_{h n}+\lambda_{s} \mu_{f n}} .
$$

The comparative static derivatives show that $\mu_{h n}$ and $\mu_{f n}$ are independent of $\lambda_{s}$. Thus, $\frac{\partial\left(v_{f o}-v_{f e}\right)}{\partial \lambda_{s}}<0$ and $\lim _{\lambda_{s} \rightarrow \infty}\left(v_{f o}-v_{f e}\right)=0$.

\section{B.2 Proof of Proposition 5}

The above comparative static derivatives with respect to $\lambda_{s}$ show that $\frac{\partial \mu_{f o}(\theta)}{\partial \lambda_{s}}>0, \frac{\partial \mu_{f e}(\theta)}{\partial \lambda_{s}}<0$, and $\frac{\partial \mu_{i}(\theta)}{\partial \lambda_{s}}=0$, for all $i \neq f o, f e$. Thus,

$$
\begin{aligned}
\frac{\partial W(\theta)}{\partial \lambda_{s}} & =\frac{\partial \mu_{h o}(\theta)}{\partial \lambda_{s}} u_{h}+\frac{\partial \mu_{l o}(\theta)}{\partial \lambda_{s}} u_{l}+\frac{\partial \mu_{f o}(\theta)}{\partial \lambda_{s}} u_{f}+\frac{\partial \mu_{f e}(\theta)}{\partial \lambda_{s}} u_{e} \\
& =\frac{\partial \mu_{f o}(\theta)}{\partial \lambda_{s}}\left(u_{f}-u_{e}\right)+\frac{\partial\left(\mu_{f o}+\mu_{f e}\right)(\theta)}{\partial \lambda_{s}} u_{e} \\
& =\frac{\partial \mu_{f o}(\theta)}{\partial \lambda_{s}}\left(u_{f}-u_{e}\right)-\frac{\partial \mu_{f n}(\theta)}{\partial \lambda_{s}} u_{e} \\
& =\frac{\partial \mu_{f o}(\theta)}{\partial \lambda_{s}}\left(u_{f}-u_{e}\right)>0 .
\end{aligned}
$$

\section{B.3 Proof of Proposition 6}

Recall from Claim 1 that the steady-state population is determined by a solution of $F\left(x ; n_{f}\right) \equiv$ $\left(F_{l o}\left(x ; n_{f}\right), F_{h n}\left(x ; n_{f}\right)\right)=0$ where

$$
\begin{aligned}
F_{l o}\left(x ; n_{f}\right) & \equiv\left(\lambda_{c} x_{2}+\lambda_{f}\left(n_{f}-g_{f n}(x)\right)\right) x_{1}+\rho_{u} x_{1}-\rho_{d}\left(n_{h}-x_{2}\right), \\
F_{h n}\left(x ; n_{f}\right) & \equiv\left(\lambda_{c} x_{1}+\lambda_{f} g_{f n}(x)\right) x_{2}+\rho_{d} x_{2}-\rho_{u}\left(n_{l}-x_{1}\right),
\end{aligned}
$$


and $g_{f n}(x) \equiv n_{a}-n_{h}+x_{2}-x_{1}$. We extend the system $F\left(x ; n_{f}\right)=0$ such that $n_{f}$ can be any real number and $x$ can be any real vector of length 2 . Each solution $x=\left(x_{1}, x_{2}\right)$ defines a vector $\mu=\left(\mu_{i}\right)_{i \in \mathcal{T}}$ as $\left(\mu_{l o}, \mu_{h n}, \mu_{l n}, \mu_{h o}\right)=\left(x_{1}, x_{2}, n_{l}-x_{1}, n_{h}-x_{2}\right)$ and $\left(\mu_{f n}, \mu_{f o}, \mu_{f e}\right)$ by (9) and (10). According to Claim 1, if $n_{f}>0$, a solution exists in certain domain (denoted by $X(\theta)$ in the claim) such that the resulting vector $\mu$ is a steady-state population. In general, without any restrictions on $n_{f}$, the vector $\mu$ may not even be positive.

The proof consists of three steps. First, for $n_{f}=0$, we find a population measure $\hat{\mu}$ such that $\hat{x} \equiv\left(\hat{\mu}_{l o}, \hat{\mu}_{h n}\right)$ solves $F\left(x ; n_{f}\right)=0$. Second, by Implicit Function Theorem, we differentiate a solution function $x\left(n_{f}\right)$ defined in the neighborhood of $n_{f}=0$ and $x=\hat{x}$, and obtain the comparative static derivative $\left.\mu_{i}^{\prime} \equiv \frac{\partial \mu_{i}}{\partial n_{f}}\right|_{n_{f}=0}$ for each $i \in \mathcal{T}$. Last, we prove the following claim:

Claim 2. There exist $\beta_{1}>0$ and $\beta_{2}$, each being independent of $\lambda_{s}$, such that

$$
\left.\frac{\partial v_{f n}}{\partial n_{f}}\right|_{n_{f}=0}=\beta_{1} \lambda_{s}+\beta_{2}
$$

Then, Proposition 6 follows immediately.

\section{B.3.1 A benchmark model $\left(n_{f}=0\right)$}

We set $\hat{\mu}_{i}=0$ for every fund type $i \in \mathcal{T}_{f}$, and impose

$$
\begin{aligned}
& \hat{\mu}_{h o}=n_{h}-\hat{\mu}_{h n}, \quad \hat{\mu}_{l o}=n_{a}-\hat{\mu}_{h o}=n_{a}-n_{h}+\hat{\mu}_{h n}, \quad \text { and } \\
& \hat{\mu}_{l n}=n_{l}-\hat{\mu}_{l o}=n_{c}-n_{a}-\hat{\mu}_{h n} .
\end{aligned}
$$

By substituting the above expressions of $\hat{\mu}_{l o}$ and $\hat{\mu}_{l n}$ in

$$
\lambda_{c} \hat{\mu}_{l o} \hat{\mu}_{h n}+\rho_{d} \hat{\mu}_{h n}-\rho_{u} \hat{\mu}_{l n}=0, \quad \mu-\mathrm{hn}
$$

we obtain

$$
\hat{\mu}_{h n}=\frac{1}{2}\left(\sqrt{\left(R+n_{a}-n_{h}\right)^{2}+4 R \cdot n_{h}\left(1-n_{a}\right)}-\left(R+n_{a}-n_{h}\right)\right),
$$


where $R \equiv \frac{\rho_{u}+\rho_{d}}{\lambda_{c}}$. It is clear that $\hat{x}=\left(\hat{\mu}_{l o}, \hat{\mu}_{h n}\right)$ solves the system $F\left(x ; n_{f}\right)=0$.

\section{B.3.2 Comparative static derivatives of $\mu$ with respect to $n_{f}$}

We apply Implicit Function Theorem. $F\left(x ; n_{f}\right)$ is an infinitely differentiable function of $x \in \mathbb{R}^{2}$ and $n_{f} \in \mathbb{R}$, and the Jacobian matrix $\nabla_{x} F(\hat{x} ; 0)$ is invertible (see Equation 11). As such, there is a differentiable function $x\left(n_{f}\right)$ defined in a neighborhood of $n_{f}=0$ and $x=\hat{x}$ such that $F\left(x\left(n_{f}\right) ; n_{f}\right)=0$. It is important to note that the derivative of $x\left(n_{f}\right)$ at any $n_{f}>0$ is independent of the choice of the function $x\left(n_{f}\right)$; Claim 1 ensures that any choice of function $x\left(n_{f}\right)$ gives the same value of $x$ for each $n_{f}>0$.

As explained above, the function $x\left(n_{f}\right)$, together with (9) and (10), defines $\mu\left(n_{f}\right)=$ $\left(\mu_{i}\left(n_{f}\right)\right)_{i \in \mathcal{T}}$, which is also differentiable. Let $\left.\mu_{i}^{\prime} \equiv \frac{\partial \mu}{\partial n_{f}}\right|_{n_{f}=0}$ for each $i \in \mathcal{T}$. Then,

$$
\begin{aligned}
{\left[\begin{array}{c}
\mu_{l o}^{\prime} \\
\mu_{h n}^{\prime}
\end{array}\right] } & =-\left[\nabla_{x} F(\hat{x} ; 0)\right]^{-1} \frac{\partial F(\hat{x} ; 0)}{\partial n_{f}} \\
& =-\left[\begin{array}{cc}
\lambda_{c} \hat{\mu}_{h n}+\lambda_{f} \hat{\mu}_{l o}+\rho_{u} & \left(\lambda_{c}-\lambda_{f}\right) \hat{\mu}_{l o}+\rho_{d} \\
\left(\lambda_{c}-\lambda_{f}\right) \hat{\mu}_{h n}+\rho_{u} & \lambda_{c} \hat{\mu}_{l o}+\lambda_{f} \hat{\mu}_{h n}+\rho_{d}
\end{array}\right]^{-1}\left[\begin{array}{c}
\lambda_{f} \hat{\mu}_{l o} \\
0
\end{array}\right] .
\end{aligned}
$$

Also, $\mu_{h o}^{\prime}=-\mu_{h n}^{\prime}$ and $\mu_{l n}^{\prime}=-\mu_{l o}^{\prime}$.

From (9),

$$
\begin{aligned}
& \mu_{f n}^{\prime}=1-\mu_{h n}^{\prime}+\mu_{l o}^{\prime} \\
& =1-\frac{1}{\operatorname{det}\left(\nabla_{x} F(\hat{x} ; 0)\right)}\left[\begin{array}{ll}
1 & -1
\end{array}\right]\left[\begin{array}{cc}
\lambda_{c} \hat{\mu}_{l o}+\lambda_{f} \hat{\mu}_{h n}+\rho_{d} & * \\
-\left(\lambda_{c}-\lambda_{f}\right) \hat{\mu}_{h n}-\rho_{u} & *
\end{array}\right]\left[\begin{array}{c}
\lambda_{f} \hat{\mu}_{l o} \\
0
\end{array}\right] \\
& =1-\frac{\lambda_{f} \hat{\mu}_{l o}\left(\lambda_{c} \hat{\mu}_{l o}+\lambda_{c} \hat{\mu}_{h n}+\rho_{d}+\rho_{u}\right)}{\operatorname{det}\left(\nabla_{x} F(\hat{x} ; 0)\right)},
\end{aligned}
$$


where

$$
\begin{aligned}
\operatorname{det}\left(\nabla_{x} F(\hat{x} ; 0)\right)= & \left(\lambda_{c} \hat{\mu}_{h n}+\lambda_{f} \hat{\mu}_{l o}+\rho_{u}\right)\left(\lambda_{c} \hat{\mu}_{l o}+\lambda_{f} \hat{\mu}_{h n}+\rho_{d}\right) \\
& -\left(\left(\lambda_{c}-\lambda_{f}\right) \hat{\mu}_{h n}+\rho_{u}\right)\left(\left(\lambda_{c}-\lambda_{f}\right) \hat{\mu}_{l o}+\rho_{d}\right) \\
= & \left(\lambda_{c} \hat{\mu}_{h n}+\rho_{u}\right)\left(\lambda_{f} \hat{\mu}_{h n}+\lambda_{f} \hat{\mu}_{l o}\right)+\left(\lambda_{f} \hat{\mu}_{h n}+\lambda_{f} \hat{\mu}_{l o}\right)\left(\lambda_{c} \hat{\mu}_{l o}+\rho_{d}\right) \\
= & \left(\lambda_{f} \hat{\mu}_{h n}+\lambda_{f} \hat{\mu}_{l o}\right)\left(\lambda_{c} \hat{\mu}_{l o}+\lambda_{c} \hat{\mu}_{h n}+\rho_{d}+\rho_{u}\right) .
\end{aligned}
$$

It follows that

$$
\mu_{f n}^{\prime}=\frac{\hat{\mu}_{h n}}{\hat{\mu}_{h n}+\hat{\mu}_{l o}}>0
$$

Next, from 10,

$$
\mu_{f o}=\frac{\left(\lambda_{f} \mu_{h n}+\lambda_{s} \mu_{f n}\right)\left(n_{f}-\mu_{f n}\right)}{\lambda_{f} \mu_{h n}+\lambda_{s} \mu_{f n}+\rho_{e}} .
$$

Thus,

$$
\begin{aligned}
& \mu_{f o}^{\prime}=\frac{\left(1-\mu_{f n}^{\prime}\right)\left(\lambda_{f} \hat{\mu}_{h n}\right)}{\rho_{e}+\lambda_{f} \hat{\mu}_{h n}}=\frac{\lambda_{f} \hat{\mu}_{h n} \hat{\mu}_{l o}}{\left(\rho_{e}+\lambda_{f} \hat{\mu}_{h n}\right)\left(\hat{\mu}_{h n}+\hat{\mu}_{l o}\right)}>0, \\
& \mu_{f e}^{\prime}=1-\mu_{f n}^{\prime}-\mu_{f o}^{\prime}=\frac{\rho_{e} \hat{\mu}_{l o}}{\left(\rho_{e}+\lambda_{f} \hat{\mu}_{h n}\right)\left(\hat{\mu}_{h n}+\hat{\mu}_{l o}\right)}>0 .
\end{aligned}
$$

\section{B.3.3 Proof of Claim 2}

From (14),

$$
r v_{f n}=\lambda_{f} \mu_{l o} g_{2}+\lambda_{s} \mu_{f e} g_{3},
$$

where $g_{2}$ and $g_{3}$ are determined by (15) and (18), respectively.

Let $\left.v_{f n}^{\prime} \equiv \frac{\partial v_{f n}}{\partial n_{f}}\right|_{n_{f}=0}$ and $\left.g_{m}^{\prime} \equiv \frac{\partial g_{m}}{\partial n_{f}}\right|_{n_{f}=0}$ for $m=2,3$. Then

$$
r v_{f n}^{\prime}=\lambda_{f}\left(\hat{g}_{2} \mu_{l o}^{\prime}+\hat{\mu}_{l o} g_{2}^{\prime}\right)+\lambda_{s}\left(\hat{g}_{3} \mu_{f e}^{\prime}+\hat{\mu}_{f e} g_{3}^{\prime}\right) .
$$

We find the value of each variable on the right-hand side of the above equation. For certain variables that we will use later, we remark whether the values are strictly positive and/or independent of $\lambda_{s}$.

We have observed the following properties: 
1. (from (23)) the population $\hat{\mu}=\left(\mu_{i}\right)_{i \in \mathcal{T}}$ is strictly positive for corporate types, zero for fund types, and independent of $\lambda_{s}$,

2. (from (24), (25), and (26)) the derivative $\mu^{\prime}$ is independent of $\lambda_{s}$,

3. (from 15) As $g_{3}=\frac{u_{f}-u_{e}}{2 r+2 \rho_{e}+\lambda_{f} \mu_{h n}+\lambda_{s} \mu_{f n}}$, we have $\hat{g}_{3}=\frac{u_{f}-u_{e}}{2 r+2 \rho_{e}+\lambda_{f} \hat{\mu}_{h n}}>0$ and $g_{3}^{\prime}=$ $-\frac{\left(\lambda_{f} \mu_{h n}^{\prime}+\lambda_{s} \mu_{f n}^{\prime}\right) \hat{g}_{3}}{2 r+2 \rho_{e}+\lambda_{f} \hat{\mu}_{h n}}$, which are independent of $\lambda_{s}$.

It remains to find the values of $\hat{g}_{2}$ and $g_{2}^{\prime}$.

To state how $g_{2}$ is determined by (18), let $c_{1} \equiv 2\left(r+\rho_{u}+\rho_{d}\right)+\lambda_{c}\left(\mu_{l o}+\mu_{h n}\right), c_{2} \equiv$ $2 \rho_{u}+\lambda_{c} \mu_{h n}$, and

$$
D \equiv\left[\begin{array}{cc}
c_{1}+\lambda_{f}\left(\mu_{f o}+\mu_{f e}\right) & c_{1}+\lambda_{f} \mu_{f n} \\
c_{2}-\lambda_{f} \mu_{h n} & c_{2}+2 r+\lambda_{f}\left(\mu_{l o}+\mu_{f n}\right)
\end{array}\right]
$$

Also, let $\alpha_{1} \equiv \frac{-D_{21}}{\operatorname{det}(D)}$ and $\alpha_{2} \equiv \frac{D_{11}}{\operatorname{det}(D)}$. Then,

$$
g_{2}=\alpha_{1}\left(u_{h}-u_{l}-\lambda_{f} \mu_{f e} g_{3}\right)+\alpha_{2}\left(u_{f}-u_{l}-2 \rho_{e} g_{3}-\lambda_{s} \mu_{f e} g_{3}\right) .
$$

Note that, when $n_{f}=0$,

$$
\begin{aligned}
\hat{c}_{1} & =2\left(r+\rho_{u}+\rho_{d}\right)+\lambda_{c}\left(\hat{\mu}_{l o}+\hat{\mu}_{h n}\right)>0, \\
\hat{c}_{2} & =2 \rho_{u}+\lambda_{c} \hat{\mu}_{h n}>0, \\
\hat{D} & =\left[\begin{array}{cc}
\hat{c}_{1} & \hat{c}_{1} \\
\hat{c}_{2}-\lambda_{f} \hat{\mu}_{h n} & \hat{c}_{2}+2 r+\lambda_{f} \hat{\mu}_{l o}
\end{array}\right] \quad \text { (with a strictly positive determinant), } \\
\hat{\alpha}_{1} & =\frac{-\hat{c}_{2}+\lambda_{f} \hat{\mu}_{h n}}{\operatorname{det}(\hat{D})}(\text { the exact value is unnecessary for our proof), and } \\
\hat{\alpha}_{2} & =\frac{\hat{c}_{1}}{\operatorname{det}(\hat{D})}=\frac{1}{2 r+\lambda_{f}\left(\hat{\mu}_{l o}+\hat{\mu}_{h n}\right)}>0,
\end{aligned}
$$

which are all independent of $\lambda_{s}$. It follows that

$$
\hat{g}_{2}=\hat{\alpha}_{1}\left(u_{h}-u_{l}\right)+\hat{\alpha}_{2}\left(u_{f}-u_{l}-2 \rho_{e} \hat{g}_{3}\right)
$$


is independent of $\lambda_{s}$.

Last, let $c_{1}^{\prime}, c_{2}^{\prime}, \alpha_{1}^{\prime}$, and $\alpha_{2}^{\prime}$ be the corresponding variables' derivatives: e.g., $\left.c_{1}^{\prime} \equiv \frac{\partial c_{1}}{\partial n_{f}}\right|_{n_{f}=0}$. The derivatives are all independent of $\lambda_{s}$, because $\hat{\mu}$ and $\mu^{\prime}$ are independent of $\lambda_{s}$. Therefore,

$$
\begin{aligned}
g_{2}^{\prime}= & \alpha_{1}^{\prime}\left(u_{h}-u_{l}-\lambda_{f} \hat{\mu}_{f e} \hat{g}_{3}\right)-\hat{\alpha}_{1} \lambda_{f}\left(\mu_{f e}^{\prime} \hat{g}_{3}+\hat{\mu}_{f e} g_{3}^{\prime}\right) \\
& +\alpha_{2}^{\prime}\left(u_{f}-u_{l}-2 \rho_{e} \hat{g}_{3}-\lambda_{s} \hat{\mu}_{f e} \hat{g}_{3}\right)-\hat{\alpha}_{2}\left(2 \rho_{e} g_{3}^{\prime}+\lambda_{s} \mu_{f e}^{\prime} \hat{g}_{3}+\lambda_{s} \hat{\mu}_{f e} g_{3}^{\prime}\right) \\
= & \alpha_{1}^{\prime}\left(u_{h}-u_{l}\right)-\hat{\alpha}_{1} \lambda_{f} \mu_{f e}^{\prime} \hat{g}_{3}+\alpha_{2}^{\prime}\left(u_{f}-u_{l}-2 \rho_{e} \hat{g}_{3}\right)-\hat{\alpha}_{2}\left(2 \rho_{e} g_{3}^{\prime}+\lambda_{s} \mu_{f e}^{\prime} \hat{g}_{3}\right) . \quad\left(\text { as } \hat{\mu}_{f e}=0\right)
\end{aligned}
$$

Only the last term $-\hat{\alpha}_{2}\left(2 \rho_{e} g_{3}^{\prime}+\lambda_{s} \mu_{f e}^{\prime} \hat{g}_{3}\right)$ is (affinely) dependent on $\lambda_{s}$, through $-\hat{\alpha}_{2} \mu_{f e}^{\prime} \hat{g}_{3} \lambda_{s}$ and $g_{3}^{\prime}=-\frac{\left(\lambda_{f} \mu_{h n}^{\prime}+\lambda_{s} \mu_{f n}^{\prime}\right) \hat{g}_{3}}{2 r+2 \rho_{e}+\lambda_{f} \hat{\mu}_{h n}}$. As such, $g_{2}^{\prime}=\gamma_{1} \lambda_{s}+\gamma_{2}$, for $\gamma_{1}=\hat{\alpha}_{2} \hat{g}_{3}\left(\frac{2 \rho_{e} \mu_{f n}^{\prime}}{2 r+2 \rho_{e}+\lambda_{f} \hat{\mu}_{h n}}-\mu_{f e}^{\prime}\right)$ and some $\gamma_{2}$ which aggregates all remaining terms. Both $\gamma_{1}$ and $\gamma_{2}$ are independent of $\lambda_{s}$. Finally,

$$
\begin{aligned}
r v_{f n}^{\prime} & =\lambda_{f}\left(\hat{g}_{2} \mu_{l o}^{\prime}+\hat{\mu}_{l o} g_{2}^{\prime}\right)+\lambda_{s}\left(\hat{g}_{3} \mu_{f e}^{\prime}+\hat{\mu}_{f e} g_{3}^{\prime}\right) \\
& =\lambda_{f}\left(\hat{g}_{2} \mu_{l o}^{\prime}+\hat{\mu}_{l o}\left(\gamma_{1} \lambda_{s}+\gamma_{2}\right)\right)+\lambda_{s} \hat{g}_{3} \mu_{f e}^{\prime} \quad\left(\text { as } \hat{\mu}_{f e}=0\right) \\
& =\left(\lambda_{f} \hat{\mu}_{l o} \gamma_{1}+\hat{g}_{3} \mu_{f e}^{\prime}\right) \lambda_{s}+\left(\lambda_{f} \hat{g}_{2} \mu_{l o}^{\prime}+\lambda_{f} \hat{\mu}_{l o} \gamma_{2}\right)
\end{aligned}
$$

where the coefficient of $\lambda_{s}$ and the last term are both independent of $\lambda_{s}$.

It remains to show that the coefficient of $\lambda_{s}$ is strictly positive:

$$
\begin{aligned}
\lambda_{f} \hat{\mu}_{l o} \gamma_{1}+\hat{g}_{3} \mu_{f e}^{\prime} & =\lambda_{f} \hat{\mu}_{l o} \hat{\alpha}_{2} \hat{g}_{3}\left(\frac{2 \rho_{e} \mu_{f n}^{\prime}}{2 r+2 \rho_{e}+\lambda_{f} \hat{\mu}_{h n}}-\mu_{f e}^{\prime}\right)+\hat{g}_{3} \mu_{f e}^{\prime} \\
& >-\lambda_{f} \hat{\mu}_{l o} \hat{\alpha}_{2} \hat{g}_{3} \mu_{f e}^{\prime}+\hat{g}_{3} \mu_{f e}^{\prime} \quad\left(\text { as } \hat{\mu}_{l o}, \hat{\alpha}_{2}, \hat{g}_{3}, \mu_{f n}^{\prime}, \hat{\mu}_{h n}\right. \text { are strictly positive) } \\
& =\mu_{f e}^{\prime} \hat{g}_{3}\left(1-\lambda_{f} \hat{\alpha}_{2} \hat{\mu}_{l o}\right) \\
& \left.=\mu_{f e}^{\prime} \hat{g}_{3}\left(1-\frac{\lambda_{f} \hat{\mu}_{l o}}{2 r+\lambda_{f}\left(\hat{\mu}_{l o}+\hat{\mu}_{h n}\right)}\right) \quad \text { (from } 27\right) \\
& >0 . \quad\left(\text { as } \mu_{f e}^{\prime} \text { and } \hat{g}_{3}\right. \text { are strictly positive) }
\end{aligned}
$$




\section{Proofs for Section 5}

For any regular environment $\theta \equiv(n, r, u, \rho, \lambda)$, we consider a sequence $\theta^{\kappa} \equiv(n, r, u, \rho, \kappa \lambda)$ with $\kappa \rightarrow \infty$. Let $\mu^{\kappa}$ be the unique steady-state solution of $P\left(\theta^{\kappa}\right)$ and $v^{\kappa}$ be the unique solution of $V\left(\theta^{\kappa}\right)$ with $\mu(t)$ being replaced by $\mu^{\kappa}$.

\section{C.1 Proof of Proposition 7}

We prove Part 1 and relegate the proof for Part 2 to the Supplemental Appendix.

In solving $P\left(\theta^{\kappa}\right)$, it is more convenient to take $z \equiv 1 / \kappa$ and define another market $\psi^{z} \equiv(n, r, u, z \rho, \lambda)$. (i.e., low type-change rates, instead of high search rates) and solve $P\left(\psi^{z}\right)$. It is easy to verify that the unique steady-state solution $\mu^{\kappa}$ of $P\left(\theta^{\kappa}\right)$ also uniquely solves $P\left(\psi^{z}\right)$. Last, define $\psi^{0} \equiv(n, r, u, 0, \lambda)$.

Lemma 4. $\mu^{0} \in \mathbb{R}^{\mathcal{T}}$ is a steady-state solution of $P\left(\psi^{0}\right)$ if and only if

1. $\left(\right.$ when $\left.n_{f}+n_{h}>n_{a}\right) \mu_{h o}^{0}=\min \left\{n_{a}, n_{h}\right\}, \mu_{h n}^{0}=n_{h}-\mu_{h o}^{0}, \mu_{l o}^{0}=0, \mu_{l n}^{0}=n_{l}, \mu_{f o}^{0}=$ $\max \left\{0, n_{a}-n_{h}\right\}, \mu_{f e}^{0}=0$, and $\mu_{f n}^{0}=n_{f}-\mu_{f o}^{0}-\mu_{f e}^{0}$.

2. $\left(\right.$ when $\left.n_{f}+n_{h}<n_{a}\right) \mu_{h o}^{0}=n_{h}, \mu_{h n}^{0}=0, \mu_{l o}^{0}=n_{a}-n_{h}-n_{f}, \mu_{l n}^{0}=n_{l}-\mu_{l o}^{0}, \mu_{f n}^{0}=0$, and $\mu_{f o}^{0}+\mu_{f e}^{0}=n_{f}$.

Note that the problem $P\left(\psi^{0}\right)$ has multiple steady-state solutions in Case $2\left(n_{f}+n_{h}<n_{a}\right)$, where many possible combinations of $\left(\mu_{f o}, \mu_{f e}\right)$ satisfy $\mu_{f o}+\mu_{f e}=n_{f}$.

(Proof) The problem $P\left(\psi^{0}\right)$ consists of

$$
\begin{aligned}
\left(\lambda_{c} \mu_{l o}+\lambda_{f} \mu_{f o}+\lambda_{f} \mu_{f e}\right) \mu_{h n} & =0, & & \text { from } \mu \text {-ho }) \\
\left(\lambda_{c} \mu_{h n}+\lambda_{f} \mu_{f n}\right) \mu_{l o} & =0, & & \text { from } \mu-\ln ) \\
\lambda_{f}\left(\mu_{l o} \mu_{f n}-\mu_{h n} \mu_{f o}\right)+\lambda_{s} \mu_{f n} \mu_{f e} & =0, & & \text { from } \mu \text {-fo })
\end{aligned}
$$

and the following four conditions that replace $\mu$-hn $, \mu-\mathrm{lo}, \mu-\mathrm{fe})$, and $(\mu-\mathrm{fn})$ :

$\mu_{h o}+\mu_{h n}=n_{h}, \quad \mu_{l o}+\mu_{l n}=n_{l}, \quad \mu_{h o}+\mu_{l o}+\mu_{f o}+\mu_{f e}=n_{a}, \quad$ and $\quad \mu_{f n}+\mu_{f o}+\mu_{f e}=n_{f}$. 
It follows from $\lambda_{c}, \lambda_{f}, \lambda_{c}>0$ that

$$
\mu_{l o} \mu_{h n}=\mu_{f o} \mu_{h n}=\mu_{f e} \mu_{h n}=\mu_{l o} \mu_{f n}=\mu_{f n} \mu_{f e}=0 .
$$

Suppose that $n_{f}+n_{h}>n_{a}$. If $\mu_{l o}>0$ or $\mu_{f e}>0$, then $\mu_{h n}=0$ and $\mu_{f n}=0$, which results in a contradiction: $\mu_{h o}+\left(\mu_{f o}+\mu_{f e}\right)=n_{h}+n_{f}>n_{a}$. As $\mu_{l o}=\mu_{f e}=0$, either $\mu_{f o}=0$ or $\mu_{h n}=0$. As $\mu_{h o}+\mu_{l o}+\mu_{f o}+\mu_{f e}=n_{a}>0$, if $\mu_{f o}=0$, then $\mu_{h o}=n_{a}$; for otherwise $\mu_{h n}=0$ implies that $\mu_{h o}=n_{h}$ and $\mu_{f o}=n_{a}-n_{h}$. On the other hand, if $n_{f}+n_{h}<n_{a}$, then $\mu_{l o}>0$, which implies that $\mu_{h n}=\mu_{f n}=0$. Thus, $\mu_{h o}=n_{h}, \mu_{f o}+\mu_{f e}=n_{f}$, and $\mu_{l o}=n_{a}-n_{h}-n_{f}$.

The following lemma implies that $\lim _{\kappa \rightarrow \infty} \mu^{\kappa}$ exists in $\mathbb{R}_{+}^{\mathcal{T}}$ :

Lemma 5. There exists a solution $\mu^{*}$ of $P\left(\psi^{0}\right)$ such that $\mu^{*} \equiv \lim _{\kappa \rightarrow \infty} \mu^{\kappa}$.

(Proof) For each $z \equiv 1 / \kappa$, let $F\left(\mu, \psi^{z}\right)$ denote the right-hand sides of the population equations $\mu$-hn $-\mu$-fe for a market $\psi^{z} \equiv(n, r, u, z \rho, \lambda)$. Define

$$
f(\mu, z) \equiv-\left\|F\left(\mu, \psi^{z}\right)\right\|
$$

where $\|\cdot\|$ denotes the Euclidean norm. It is clear that $\mu^{\kappa}$ with $\kappa=1 / z$ is the unique maximizer of $f$ with the maximum value equals zero. Let $M(z) \equiv\left\{\mu^{1 / z}\right\}$.

We similarly define $F\left(\mu, \psi^{0}\right)$ as the right-hand sides of the population equations for the market $\psi^{0}$ and $f(\mu, 0)$. Let $M(0)$ be the solution set of $\max _{\mu} f(\mu, 0)$. According to Lemma 4, the solution set $M(0)$ is singleton if $n_{h}+n_{f}<n_{a}$; for otherwise, $M(0)$ contains multiple solutions, each being different from others only in $\left(\mu_{f o}, \mu_{f e}\right)$ under the constraint $\mu_{f o}+\mu_{f e}=n_{f}$.

The function $f$ is continuous in $\mu$ and $z$ because the equations $F$ are continuous. Thus, by Berge's Maximum Theorem, the correspondence $M(\cdot)$ is upper hemicontinuous at $z=0$ :

1. (when $\left.n_{h}+n_{f}<n_{a}\right) \mu^{\kappa}$ converges to the unique solution of $P\left(\psi^{0}\right)$.

2. (when $n_{h}+n_{f}>n_{a}$ ) for each type $i \neq f o, f e$, the population $\mu_{i}^{\kappa}$ converges to $\mu_{i}^{0}$ given in Lemma 4, and $\mu_{f o}^{\kappa}+\mu_{f e}^{\kappa}$ converges to $n_{f}$. 
It remains to show that, when $n_{h}+n_{f}>n_{a}$, the sequence $\mu_{f e}^{\kappa}$ converges. (The convergence of $\mu_{f_{o}}^{\kappa}$ follows immediately from $\lim _{\kappa \rightarrow \infty}\left(\mu_{f o}^{\kappa}+\mu_{f e}^{\kappa}\right)=n_{f}$.)

For every $\kappa>0, \kappa\left(\lambda_{f} \mu_{h n}^{\kappa}+\lambda_{s} \mu_{f n}^{\kappa}\right) \mu_{f e}^{\kappa}=\rho_{e}\left(n_{f}-\mu_{f n}^{\kappa}-\mu_{f e}^{\kappa}\right)$ (from $\mu$-fe $)$, or equivalently that

$$
\mu_{f e}^{\kappa}=\frac{\rho_{e}\left(n_{f}-\mu_{f n}^{\kappa}\right)}{\rho_{e}+\kappa\left(\lambda_{f} \mu_{h n}^{\kappa}+\lambda_{s} \mu_{f n}^{\kappa}\right)} .
$$

We find $\lim _{\kappa \rightarrow \infty} \kappa\left(\lambda_{f} \mu_{h n}^{\kappa}+\lambda_{s} \mu_{f n}^{\kappa}\right.$ from

$$
\begin{array}{lr}
\kappa \mu_{h n}^{\kappa}\left(\lambda_{c} \mu_{l o}^{\kappa}+\lambda_{f} \mu_{f o}^{\kappa}+\lambda_{f} \mu_{f e}^{\kappa}\right)=-\rho_{d} \mu_{h n}^{\kappa}+\rho_{u} \mu_{l n}^{\kappa}, & (\text { from } \mu-\mathrm{hn}) \\
\kappa\left(\lambda_{c} \mu_{h n}^{\kappa}+\lambda_{f} \mu_{f n}^{\kappa}\right) \mu_{l o}^{\kappa}=-\rho_{u} \mu_{l o}^{\kappa}+\rho_{d} \mu_{h o}^{\kappa} . & (\text { from } \mu-\mathrm{lo})
\end{array}
$$

By the convergence of $\mu_{i}^{\kappa}$ for $i \neq f o, f e$, and the convergence of $\mu_{f e}^{\kappa}+\mu_{f o}^{\kappa}$ to $n_{f}$,

$$
\begin{aligned}
& \lim _{\kappa \rightarrow \infty} \kappa \mu_{h n}^{\kappa}=\frac{\rho_{u} \mu_{l n}^{*}}{\lambda_{c} \mu_{l o}^{*}+\lambda_{f} n_{f}}, \quad \text { and } \\
& \lim _{\kappa \rightarrow \infty} \kappa\left(\lambda_{c} \mu_{h n}^{\kappa}+\lambda_{f} \mu_{f n}^{\kappa}\right)=\frac{\rho_{d} \mu_{h o}^{*}-\rho_{u} \mu_{l o}^{*}}{\mu_{l o}^{*}}=\frac{\rho_{d} n_{h}-\rho_{u} \mu_{l o}^{*}}{\mu_{l o}^{*}}=\frac{\rho_{u} \mu_{l n}^{*}}{\mu_{l o}^{*}} .
\end{aligned}
$$

It follows that

$$
\begin{aligned}
\lim _{\kappa \rightarrow \infty} \kappa\left(\lambda_{f} \mu_{h n}^{\kappa}+\lambda_{s} \mu_{f n}^{\kappa}\right) & =\frac{\lambda_{s} \rho_{u} \mu_{l n}^{*}}{\lambda_{f} \mu_{l o}^{*}}+\left(\lambda_{f}-\frac{\lambda_{c} \lambda_{s}}{\lambda_{f}}\right) \frac{\rho_{u} \mu_{l n}^{*}}{\lambda_{c} \mu_{l o}^{*}+\lambda_{f} n_{f}} \\
& =\frac{\rho_{u} \mu_{l n}^{*}}{\mu_{l o}^{*}} \frac{\lambda_{f} \mu_{l o}^{*}+\lambda_{s} n_{f}}{\lambda_{c} \mu_{l o}^{*}+\lambda_{f} n_{f}}>0 .
\end{aligned}
$$

Therefore,

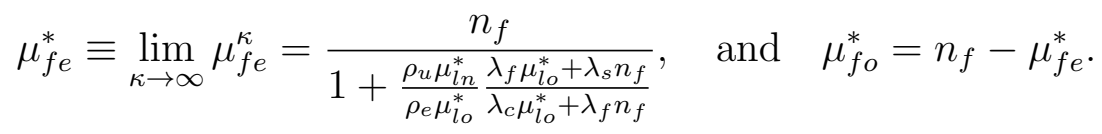

\section{C.2 Proof of Proposition 8}

By Lemma 4 and Lemma 5. if $n_{a}<n_{h}+n_{f}$, then $\mu_{h o}^{*}=\min \left\{n_{a}, n_{h}\right\}, \mu_{f o}^{*}=\max \left\{0, n_{a}-n_{h}\right\}$, $\mu_{f e}^{*}=0$, and $\mu_{l o}^{*}=0$. Since $\mu^{*}$ coincides with the efficient asset allocation $\bar{\mu}$, we have 
$W^{*}=\bar{W}$. The independence of $W^{*}$ on $u_{f}$ and $u_{e}$ is trivial as $\mu_{f o}^{*}=\mu_{f e}^{*}=0$. The independent of $W^{*}$ on $\lambda_{c}$ also follows from $\bar{W}$ 's independence of any search friction. When $n_{h}<n_{a}<n_{h}+n_{f}$, we have $\mu_{f o}>0$, so $W^{*}=\bar{W}$ is strictly increasing in $u_{f}$.

If $n_{a}>n_{h}+n_{f}$, then $W^{*}=\bar{W}-\mu_{f e}^{*}\left(u_{f}-u_{e}\right)$. We have $W^{*}<\bar{W}$ because

$$
\mu_{f e}^{*}=\frac{n_{f}}{1+\frac{\rho_{u} \mu_{l n}^{*}}{\rho_{e} \mu_{l o}^{*}} \frac{\lambda_{f} \mu_{l o}^{*}+\lambda_{s} n_{f}}{\lambda_{c} \mu_{l o}^{*}+\lambda_{f} n_{f}}}>0,
$$

The welfare $W^{*}$ is increasing in $u_{f}$ and $u_{e}$ as $\mu_{f o}^{*}$ and $\mu_{f e}^{*}$ are strictly positive. Moreover,

$\mu_{f e}^{*}$ is decreasing in $\lambda_{s}$ and increasing in $\lambda_{c}$. Thus, the welfare $W^{*}$ is increasing in $\lambda_{s}$ and decreasing in $\lambda_{c}$.

\section{References}

Ahern, K. R. (2012): "Bargaining power and industry dependence in mergers," Journal of Financial Economics, 103(3), 530-550.

Allen, F., And A. Babus (2009): "Networks in finance," The Network Challenge, pp. $367-382$.

Aminud, Y., H. Mendelson, and L. H. Pedersen (2006): "Liquidity and asset prices," Foundations and Trends@ in Finance, 1(4), 269-364.

Andrade, G., and S. N. Kaplan (1998): "How costly is financial (not economic) distress? Evidence from highly leveraged transactions that became distressed," The Journal of Finance, 53(5), 1443-1493.

Arcot, S., Z. Fluck, J.-M. Gaspar, and U. Hege (2015): "Fund managers under pressure: Rationale and determinants of secondary buyouts," Journal of Financial Economics, 115(1), 102-135.

Atkeson, A. G., A. L. Eisfeldt, and P.-O. Weill (2015): "Entry and exit in OTC derivatives markets," Econometrica, 83(6), 2231-2292. 
Bargeron, L. L., F. P. Schlingemann, R. M. Stulz, and C. J. Zutter (2008): "Why do private acquirers pay so little compared to public acquirers?," Journal of Financial Economics, 89(3), 375-390.

Betton, S., B. E. Eckbo, and K. S. Thorburn (2008): "Corporate takeovers," Handbook of corporate finance: Empirical corporate finance, 2, 291-430.

Boone, A. L., And J. H. Mulherin (2007): "How are firms sold?," The Journal of Finance, 62(2), 847-875.

Cochrane, J. H. (2005): "The risk and return of venture capital," Journal of financial economics, $75(1), 3-52$.

DAVID, J. (2017): "The aggregate implications of mergers and acquisitions," Working Paper.

Degeorge, F., J. Martin, and L. Phalippou (2016): "On secondary buyouts," Journal of Financial Economics, 120(1), 124-145.

Duffie, D., N. GÂrleanu, and L. H. Pedersen (2005): "Over-the-Counter Markets," Econometrica, 73(6), 1815-1847.

Eisfeldt, A. L., ANd A. A. RAmpini (2006): "Capital reallocation and liquidity," Journal of monetary Economics, 53(3), 369-399.

(2008): "Managerial incentives, capital reallocation, and the business cycle," Journal of Financial Economics, 87(1), 177-199.

Farboodi, M., G. Jarosch, and R. Shimer (2017): "The Emergence of Market Structure," Working Paper 23234, National Bureau of Economic Research.

Gofman, M. (2014): "A Network-Based Analysis of Over-the-Counter Markets," Working Paper.

Gompers, P., And J. Lerner (2000): "Money chasing deals? The impact of fund inflows on private equity valuation," Journal of Financial Economics, 55(2), 281-325. 
Harris, R. S., T. Jenkinson, and S. N. Kaplan (2014): "Private equity performance: What do we know?," The Journal of Finance, 69(5), 1851-1882.

Hirsch, M. W. (2012): Differential topology, vol. 33. Springer Science \& Business Media.

Hirsch, M. W., AND S. Smale (1973): Differential equations, dynamical systems and linear algebra. Academic Press College Division.

Hochberg, Y. V., A. Luungqvist, And Y. Lu (2007): "Whom you know matters: Venture capital networks and investment performance," The Journal of Finance, 62(1), 251-301.

Hugonnier, J., B. Lester, and P.-O. Weill (2018): "Frictional intermediation in over-the-counter markets," Discussion paper, National Bureau of Economic Research.

Jensen, M. C. (1991): "Eclipse of the Public Corporation," in In The Law of Mergers, Acquisitions, and Reorganizations.

Jovanovic, B., And P. L. Rousseau (2002): "The Q-theory of mergers," American Economic Review, 92(2), 198-204.

Kaplan, S. N., And A. SchoAR (2005): "Private equity performance: Returns, persistence, and capital flows," The Journal of Finance, 60(4), 1791-1823.

Koeplin, J., A. Sarin, And A. C. Shapiro (2000): "The private company discount," Journal of Applied Corporate Finance, 12(4), 94-101.

Metrick, A., And A. Yasuda (2010): "The economics of private equity funds," The Review of Financial Studies, 23(6), 2303-2341.

Muscarella, C. J., and M. R. Vetsuypens (1990): "Efficiency and organizational structure: A study of reverse LBOs," The Journal of Finance, 45(5), 1389-1413.

Nadauld, T. D., B. A. Sensoy, K. Vorkink, and M. S. Weisbach (2016): "The liquidity cost of private equity investments: Evidence from secondary market transactions," Discussion paper, National Bureau of Economic Research. 
Neklyudov, A. (2012): "Bid-ask spreads and the over-the-counter interdealer markets: Core and peripheral dealers," Discussion paper, Working Paper HEC Lausanne.

Nosal, E., and G. Rocheteau (2011): Money, payments, and liquidity. MIT press.

Nosal, E., Y.-Y. Wong, and R. Wright (2016): "Who Wants to be a Middleman?," Discussion paper, Mimeo, University of Wisconsin Madison.

Opler, T. C. (1992): "Operating performance in leveraged buyouts: Evidence from 19851989," Financial Management, pp. 27-34.

Pagano, M., And P. Volpin (2012): "Securitization, transparency, and liquidity," The Review of Financial Studies, 25(8), 2417-2453.

Phalippou, L., and O. Gottschalg (2008): "The performance of private equity funds," The Review of Financial Studies, 22(4), 1747-1776.

Phillips, G. M., And A. Zhdanov (2017): "Venture Capital Investments and Merger and Acquisition Activity Around the World," Discussion paper, National Bureau of Economic Research.

Rhodes-Kropf, M., and D. T. Robinson (2008): "The market for mergers and the boundaries of the firm," The Journal of Finance, 63(3), 1169-1211.

Rhodes-Kropf, M., D. T. Robinson, and S. Viswanathan (2005): "Valuation waves and merger activity: The empirical evidence," Journal of Financial Economics, 77(3), 561-603.

Rubinstein, A., and A. Wolinsky (1987): "Middlemen," The Quarterly Journal of Economics, 102(3), 581-593.

SilbeR, W. L. (1991): "Discounts on restricted stock: The impact of illiquidity on stock prices," Financial analysts journal, pp. 60-64.

Simsek, A., A. Ozdaglar, and D. Acemoglu (2007): "Generalized Poincare-Hopf theorem for compact nonsmooth regions," Mathematics of Operations Research, 32(1), 193214. 
Sorensen, M., And R. Jagannathan (2015): "The public market equivalent and private equity performance," Financial Analysts Journal, 71(4), 43-50.

Trejos, A., and R. Wright (2016): "Search-based models of money and finance: An integrated approach," Journal of Economic Theory, 164, 10-31.

UsLu, S. (2016): "Pricing and liquidity in decentralized asset markets," Working Paper.

Vayanos, D., And P.-O. Weill (2008): "A search-based theory of the on-the-run phenomenon," The Journal of Finance, 63(3), 1361-1398.

WANG, Y. (2012): "Secondary buyouts: Why buy and at what price?," Journal of Corporate Finance, 18(5), 1306-1325.

Weill, P.-O. (2007): "Leaning against the wind," The Review of Economic Studies, 74(4), $1329-1354$. 